\title{
Vehicle Dynamics Approach to Driver Warning
}

\author{
Youssef A. Ghoneim \\ Research and Development Center, General Motors Corporation, 30500 Mound Road, Warren, MI 48090, USA \\ Correspondence should be addressed to Youssef A. Ghoneim; youssef.ghoneim@gm.com
}

Received 12 November 2012; Accepted 4 January 2013

Academic Editor: Martin Reisslein

Copyright (c) 2013 Youssef A. Ghoneim. This is an open access article distributed under the Creative Commons Attribution License, which permits unrestricted use, distribution, and reproduction in any medium, provided the original work is properly cited.

\begin{abstract}
This paper discusses a concept for enhanced active safety by introducing a driver warning system based on vehicle dynamics that predicts a potential loss of control condition prior to stability control activation. This real-time warning algorithm builds on available technologies such as the Electronic Stability Control (ESC). The driver warning system computes several indices based on yaw rate, side-slip velocity, and vehicle understeer using ESC sensor suite. An arbitrator block arbitrates between the different indices and determines the status index of the driving vehicle. The status index is compared to predetermined stability levels which correspond to high and low stability levels. If the index exceeds the high stability level, a warning signal (haptic, acoustic, or visual) is issued to alert the driver of a potential loss of control and ESC activation. This alert will remain in effect until the index is less than the low stability level at which time the warning signal will be terminated. A vehicle speed advisory algorithm is integrated with the warning algorithm to provide a desired vehicle speed of a vehicle traveling on a curve. Simulation results and vehicle tests were conducted to illustrate the effectiveness of the warning algorithm.
\end{abstract}

\section{Introduction}

Freeway entrance and exit ramp interchanges are the sites of far more crashes per mile driven than other segments of interstate highways. Crashes most common on exit rampsrun-off-road crashes-frequently occurred when vehicles were exiting interstates at night, in bad weather, or on curved portions of ramps. When the vehicle is driving under these conditions at a higher speed than the surface can allow, the understeer gradient of the vehicle can increase causing the vehicle to plow or decrease and becomes negative causing the vehicle to spinout.

In recent years, electronic stability control systems for motor vehicles have become increasingly popular [1-12]. Conventionally, such systems monitor vehicle stabilityrelated quantities such as a yaw rate error, that is, a deviation between yaw rates expected based on vehicle speed and steering wheel angle and an observed yaw rate, and selectively brake the wheels at one side of the vehicle in order to assist cornering, that is, to decrease the deviation between expected and observed yaw rates.

Electronic Stability Control (ESC) helps keep the vehicle on its steered path during a turn, to avoid sliding or skidding. It uses a computer linked to a series of sensors-detecting wheel speed, steering angle, yaw rate and lateral acceleration of the vehicle. During normal driving, ESC works in the background and continuously monitors steering and vehicle direction. It compares the driver's intended direction (determined through the measured steering wheel angle) to the vehicle's actual direction (determined through measured lateral acceleration, vehicle rotation (yaw), and individual road wheel speeds). ESC intervenes only when it detects a probable loss of steering control, that is, when the vehicle is not going where the driver is steering. This may happen, for example, when skidding during emergency evasive swerves, understeer or oversteer during poorly judged turns on slippery roads, or hydroplaning. If the vehicle starts to drift, the system momentarily brakes one or more wheels and, depending on the system, reduces engine power to keep the car on the steered course. However, Electronic Stability Control cannot override the laws of physics. If a driver exceeds the friction capabilities of the road surface, ESC cannot prevent a crash. It is a tool to help the driver maintain control.

In this paper, we introduce a driver warning algorithm integrated with the Electronic Stability Control system. The warning system is designed to further assist a driver by warning of an impending ESC activation so that the driver will reduce the vehicle's speed prior to the need for ESC 


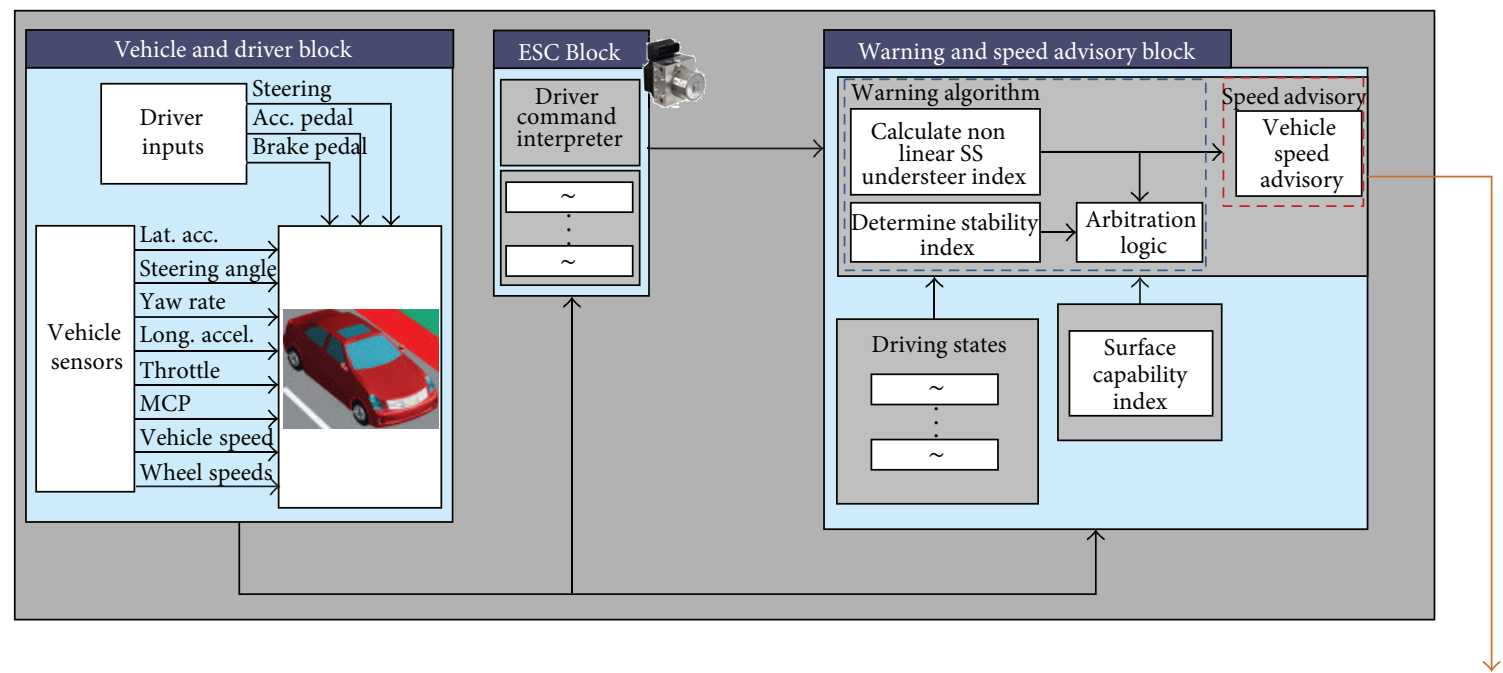

Driver warning index
(i) Haptic warning
(ii) Text message
(iii) Chime
(iv) $\cdots$
Vehicle speed advisory
(i) Text message

FIGURE 1: Schematic diagram of the warning algorithm.

intervention. It is hoped that the warning system will assist the driver in recognizing and avoiding instances of potential loss of vehicle control and also reduce usage of ESC.

The body of the paper begins first with system architecture including a brief description of the different blocks used in the warning algorithm. Second, we develop the warning signal command for both the transient and the steady-state modes of the vehicle. Third, we calculate a vehicle advisory speed in a curve based on vehicle understeer gradient. Finally, we present simulation results and vehicle tests.

\section{Warning Algorithm Architecture}

Figure 1 illustrates the schematic diagram of the warning algorithm which consists of three major parts:

(1) the vehicle and driver block which contains the following:

(i) driver inputs (steering, accelerator pedal, and brake pedal),

(ii) vehicle sensors (lateral acceleration, yaw rate, wheel speeds to estimate the vehicle longitudinal speed, throttle position, and master cylinder pressure sensor);

(2) the ESC block:

(i) command Interpreter block;

(3) the warning and speed advisory block:

(i) the driving states, (ii) the Lateral Surface Capability Index,

(iii) the warning Algorithm:

(a) the understeer index,

(b) the stability index,

(c) the arbitration logic,

(iv) the vehicle speed advisory.

\subsection{The ESC Block}

2.1.1. The Command Interpreter Block. Vehicle Yaw-Plane Dynamics. In this section, we describe the equations of dynamics for the yaw-plane motion of a vehicle. While a vehicle is undergoing handling maneuvers, it not only incurs a yaw motion, it also experiences a side-slip motion at the same time. The yaw-plane dynamics determine the performance of vehicle yaw motion characterized by vehicle yaw rate, as well as the lateral motion characterized by sideslip velocity.

The vehicle yaw-plane dynamics can be described by a second-order state [7]

$$
\left[\begin{array}{c}
\dot{v}_{y} \\
\ddot{\psi}
\end{array}\right]=\left[\begin{array}{ll}
a_{11} & a_{12} \\
a_{21} & a_{22}
\end{array}\right]\left[\begin{array}{l}
v_{y} \\
\dot{\psi}
\end{array}\right]+\left[\begin{array}{l}
b_{1} \\
b_{2}
\end{array}\right] \delta,
$$

where $v_{y}$ and $\dot{\psi}$ are the vehicle side-slip velocity (defined as the component of the vehicle velocity vector in the $y$ direction [13]) and yaw rate (defined as vehicle's angular velocity around its vertical axis), respectively, and the system coefficients, $a_{i j}$ 's and $b_{i}$ 's, are functions of vehicle mass, vehicle speed, vehicle inertia, and the front and rear cornering 
stiffness, and inevitably, the location of vehicle center of gravity are described by the parameters $a$ and $b$ :

$$
\begin{gathered}
a_{11}=-\frac{C_{f}+C_{r}}{M_{v} v_{x}} \quad a_{12}=\frac{-a C_{f}+b C_{r}}{M_{v} v_{x}}-v_{x}, \\
a_{21}=\frac{-a C_{f}+b C_{r}}{I_{z} v_{x}} \quad a_{22}=-\frac{a^{2} C_{f}+b^{2} C_{r}}{I_{z} v_{x}}, \\
b_{1}=\frac{C_{f}}{M_{v}} \quad b_{2}=\frac{a C_{f}}{I_{z}} .
\end{gathered}
$$

The transfer functions from the steering input to the vehicle yaw rate and side-slip velocity can be derived from the state

$$
\begin{aligned}
& \frac{\dot{\psi}(s)}{\delta(s)}=\frac{b_{2} s+\left(a_{21} b_{1}-a_{11} b_{2}\right)}{s^{2}-\left(a_{11}+a_{22}\right) s+\left(a_{11} a_{22}-a_{12} a_{21}\right)}, \\
& \frac{v_{y}(s)}{\delta(s)}=\frac{b_{1} s+\left(a_{12} b_{2}-a_{22} b_{1}\right)}{s^{2}-\left(a_{11}+a_{22}\right) s+\left(a_{11} a_{22}-a_{12} a_{21}\right)} .
\end{aligned}
$$

Equations (4) and (5) can be used to design a closedloop control system for vehicle stability enhancement by regulating the measured vehicle performance to its desired value determined in the following section.

Desired Vehicle Response. The desired yaw rate and the sideslip velocity commands are determined by the desired vehicle response to the driver's steering input. There are mainly two approaches of implementation: (a) using the state in (1) to perform real-time integration or (b) using transfer functions such as (4) and (5) which consist of the steady-state value of the desired yaw rate and side-slip velocity and a dynamic filter representing the desired vehicle dynamics.

When the state approach is employed to generate the commands, the desired vehicle side-slip velocity and desired yaw rate are computed based on the system differentials using nominal values of system parameters defined in (1)-(5).

When the state equation approach is employed to generate the commands, the desired vehicle side-slip velocity and desired yaw rate are computed based on the system differential equations using nominal values of system parameters:

$$
\left[\begin{array}{l}
\dot{v}_{y d} \\
\ddot{\psi}_{d}
\end{array}\right]=\left[\begin{array}{ll}
a_{11} & a_{12} \\
a_{21} & a_{22}
\end{array}\right]\left[\begin{array}{l}
v_{y d} \\
\dot{\psi}_{d}
\end{array}\right]+\left[\begin{array}{l}
b_{1} \\
b_{2}
\end{array}\right] \delta .
$$

In this reference model, the $C_{f}$ and $C_{r}$ are replaced with constants $C_{f 0}$ and $C_{r 0}$ representing the values of a highcoefficient condition on dry surface. Integration of (1) results in a desired time trace of vehicle side-slip velocity and yaw rate.

The transfer-function approach for obtaining the desired vehicle response is based on the structure of the system input-output transfer function derived in (4) and (5), but substituting the system coefficients with the nominal values forms the first-stage command:

$$
\begin{aligned}
& \frac{v_{y d}(s)}{\delta(s)}=\frac{b_{1} s+\left(a_{12} b_{2}-a_{22} b_{1}\right)}{s^{2}-\left(a_{11}+a_{22}\right) s+\left(a_{11} a_{22}-a_{12} a_{21}\right)}, \\
& \frac{\dot{\psi}_{d}(s)}{\delta(s)}=\frac{b_{2} s+\left(a_{21} b_{1}-a_{11} b_{2}\right)}{s^{2}-\left(a_{11}+a_{22}\right) s+\left(a_{11} a_{22}-a_{12} a_{21}\right)} .
\end{aligned}
$$

Equations (6) are mathematically equivalent to (5). The practical difference is that the desired natural frequency and damping ratio determined by (6) can be specified without regard to their original formation derived from the vehicle parameters.

Therefore, rewriting (6) in terms of system natural frequency and damping ratio yields

$$
\begin{aligned}
& \frac{v_{y d}(s)}{\delta(s)}=\frac{\left(s / z_{v}+1\right) \omega_{n}^{2}}{s^{2}+2 \zeta \omega_{n} s+\omega_{n}^{2}} V_{y \text { dss_gain }} \\
& \frac{\dot{\psi}_{d}(s)}{\delta(s)}=\frac{\left(s / z_{\dot{\psi}}+1\right) \omega_{n}^{2}}{s^{2}+2 \zeta \omega_{n} s+\omega_{n}^{2}} \dot{\psi}_{\text {dss_gain }},
\end{aligned}
$$

where

$$
\begin{gathered}
V_{y \text { dss_gain }}=\frac{\left(a_{12} b_{2}-v_{x}\right)-a_{22} b_{1}}{a_{12} a_{22}-a_{12} a_{21}}, \\
\dot{\psi}_{\text {dss_gain }}=\frac{a_{21} b_{1}-a_{11} b_{2}}{a_{12} a_{22}-a_{12} a_{21}}
\end{gathered}
$$

are the steady-state gains of desired steady-state side-slip velocity and yaw rate. The damping ratio and natural frequency of the desired vehicle performance can be expressed in terms of system parameters

$$
\begin{gathered}
\omega_{n}=\sqrt{a_{11} a_{22}-a_{12} a_{21}}, \\
\zeta=-\frac{a_{11}+a_{22}}{2 \omega_{n}}
\end{gathered}
$$

and tabulated as functions of vehicle speed in control software. The variables $z_{v}$ and $z_{\dot{\psi}}$ are the negative of the system zeroes for both side slip velocity and yaw rate and can be represented by

$$
\begin{gathered}
z_{v}=\left(a_{12}-v_{x}\right) \frac{b_{2}}{b_{1}}-a_{22}, \\
z_{\dot{\psi}}=a_{21} \frac{b_{2}}{b_{1}}-a_{22} .
\end{gathered}
$$

The steady-state value of the vehicle side-slip velocity can be obtained by multiplying the gain with the steering angle; that is,

$$
V_{y \text { dss }}=V_{y \text { dss_gain }} \delta .
$$


Substituting expressions in (3) and (8) into (13) yields

$$
\begin{aligned}
V_{y \mathrm{dss}} & =\frac{v_{x}\left((a+b) b C_{f} C_{r}-a C_{f} M_{v} v_{x}^{2}\right)}{(a+b)^{2} C_{f} C_{r}+M_{v} v_{x}^{2}\left(-a C_{f}+b C_{r}\right)} \delta \\
& =\left(\frac{v_{x} \delta}{(a+b)+K_{u} v_{x}^{2}}\right)\left(b-\frac{a}{a+b} \frac{M_{v} v_{x}^{2}}{C_{r}}\right),
\end{aligned}
$$

where

$$
K_{u}=\frac{M_{v}}{a+b}\left(\frac{b}{C_{f}}-\frac{a}{C_{r}}\right) .
$$

Equation (14) is composed of a product of two terms where the first term represents the steady-state of desired yaw rate. From this fact, the steady-state value of desired side-slip velocity can be implemented in terms of steady-state desired yaw rate

$$
V_{y \mathrm{~d} s \mathrm{~s}}=\dot{\psi}_{\mathrm{dss}}\left(b-\frac{a}{a+b} \frac{M_{v} v_{x}^{2}}{C_{r}}\right) .
$$

The steady-state desired yaw rate $\dot{\psi}_{\text {dss }}$ during control computation can be obtained using the following:

$$
\dot{\psi}_{\mathrm{dss}}=\frac{v_{x} \delta}{(a+b)+K_{u} v_{x}^{2}},
$$

where $K_{u}$ is obtained from computation of (15).

Therefore, (14), (15), and (16) can be used to obtain the value of $V_{y \text { dss }}$.

Given the steady-state side-slip velocity, the next step is to process such value through a dynamic filter with desired damping ratio and natural frequency representative of the system dynamics described in (7) and (8). But one question remains regarding to the significance of the non-minimumphase zero in the implementation of the side slip command.

The dynamic filter with desired damping ratio, natural frequency, and zero can be implemented using a set of two first-order differentials

$$
\begin{aligned}
& \dot{x}_{1}=V_{y \mathrm{dss}}-2 \zeta \omega_{n} x_{1}-\omega_{n}^{2} x_{2}, \\
& \dot{x}_{2}=x_{1}, \\
& v_{y d}=\omega_{n}^{2}\left(x_{2}+\frac{x_{1}}{z_{v}}\right)
\end{aligned}
$$

where $z_{v}$ is expressed in (11) and implemented in a lookup table as a function of vehicle speed. A similar second-order dynamic filter can be implemented in control computation to provide the desired yaw-rate command. Consider

$$
\begin{aligned}
& \dot{x}_{1}=\dot{\psi}_{y \mathrm{dss}}-2 \zeta \omega_{n} x_{1}-\omega_{n}^{2} x_{2}, \\
& \dot{x}_{2}=x_{1}, \\
& v_{y d}=\omega_{n}^{2}\left(x_{2}+\frac{x_{1}}{z_{\dot{\psi}}}\right) .
\end{aligned}
$$

Equations (18) and (19), which take separate desired steady-state values as the inputs, can be combined into one set of dynamic filters with a common input. Dynamics are imparted to steering wheel angle through a secondorder filter with two states. Desired yaw rate and lateral velocity are computed based on linear combinations of these states. Steady-state yaw gain, as mentioned before, can be implemented in a table look-up to account for variation of understeer coefficient when the vehicle is operated at the limit of tire adhesion. Steady state lateral gain is computed from the steady-state yaw gain through kinematics relationships. Both filter natural frequency and damping ratio are functions of vehicle velocity and are implemented as look-up tables.

The dynamic relationship between the steering angle and desired side-slip velocity can be expressed into the following form:

$$
\frac{v_{y d}(s)}{\delta(s)}=\frac{b_{1} s+a_{12} b_{2}-a_{22} b_{1}}{s^{2}+2 \zeta \omega_{n} s+\omega_{n}^{2}} .
$$

Using this relationship, a dynamic filter can be established with steering angle as input:

$$
\begin{aligned}
& \dot{x}_{1}=\delta-2 \zeta \omega_{n} x_{1}-\omega_{n}^{2} x_{2}, \\
& \dot{x}_{2}=x_{1},
\end{aligned}
$$

where $\delta=$ steering wheel angle, $\zeta=$ damping ratio, function of vehicle speed, and $\omega_{n}=$ natural frequency, function of vehicle speed.

Defining

$$
\begin{aligned}
& V_{y \text { gain }}=\Omega_{\text {gain }} g_{r}\left(b-\frac{a M_{v}}{a+b} \frac{V_{x}^{2}}{C_{r}}\right) \\
& \Omega_{\text {gain }}=\text { the yaw gain from } 3-\mathrm{d} \text { table, } \\
& g_{r}=\text { steering ratio, }
\end{aligned}
$$

we can compute for both the dynamic desired side-slip velocity and yaw rate using the two states $x_{1}$ and $x_{2}$ of a single dynamic filter described in (21):

$$
\begin{gathered}
\dot{\psi}_{\mathrm{des}}=\left[\frac{a}{I_{z}}\right] C_{f} x_{1}+\Omega_{\mathrm{gain}} g_{r} \omega_{n}^{2} x_{2}, \\
V_{y \text { des }}=\left[\frac{1}{M_{v}}\right] C_{f} x_{1}+V_{y \text { gain }} \omega_{n}^{2} x_{2} .
\end{gathered}
$$

A block diagram of the command interpreter block is illustrated in Figure 2.

\subsection{The Warning and Speed Advisory Block}

2.2.1. The Driving States. This block reads all available sensor signals, namely, the lateral and longitudinal accelerations, steering wheel angle, and yaw rate to detect the current driving situation. This block distinguishes 11 different driving modes such as cruising, braking, cornering, transient, low speed maneuvering, and reversing. 


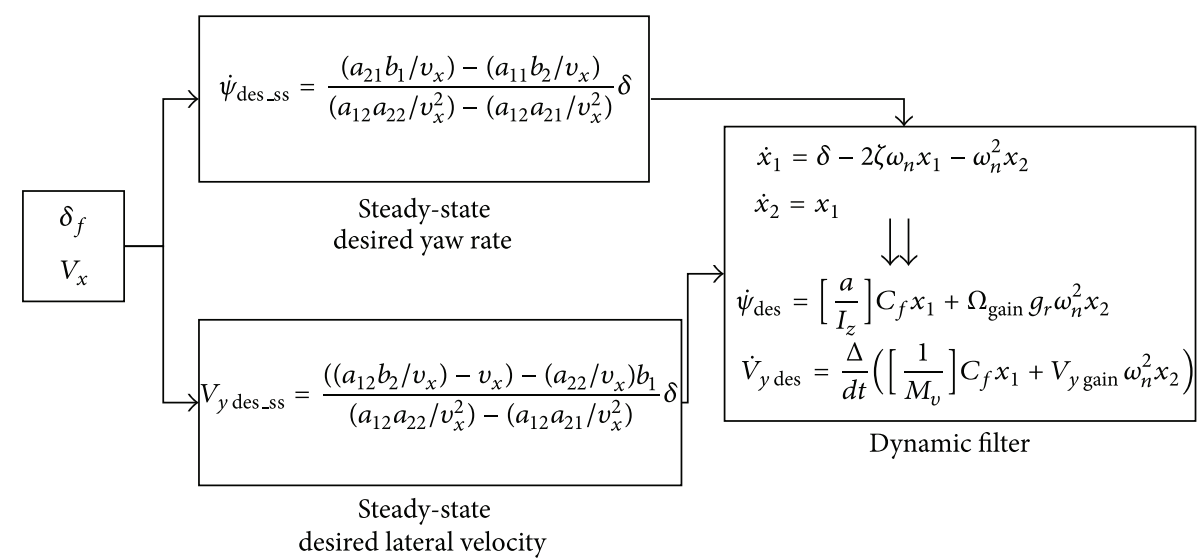

FIGURE 2: Block diagram of the command interpreter algorithm.

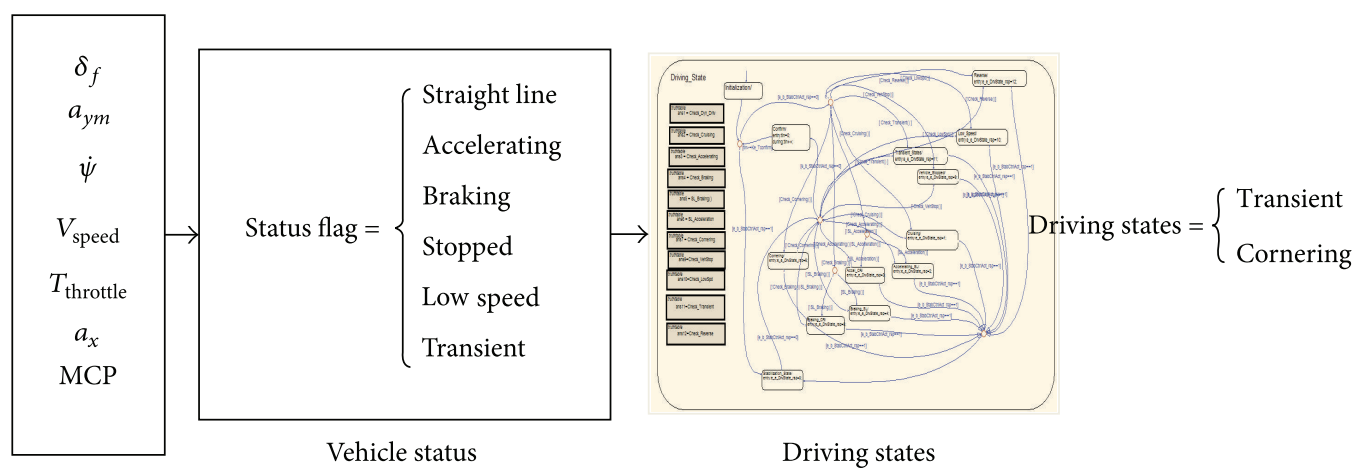

FIGURE 3: Block diagram of the driving states algorithm.

For the purpose of this study, it is sufficient to distinguish between a transient mode and a steady turn for both linearized and nonlinear relations between lateral tire forces and slip angles. We define four intermediate flags $F_{i_{\mathrm{tr}}}$, $i=1,4$.

The transient mode is determined as follows:

$\ddot{\psi}\left\{\begin{array}{l}|\ddot{\psi}|>\ddot{\psi}_{\text {trans2_th }}\left(V_{x}\right) \longrightarrow F_{1_{-} \text {tr }}=\text { true, } \\ |\ddot{\psi}| \leq \ddot{\psi}_{\text {trans_th min }}\left(V_{x}\right) \longrightarrow F_{1_{-} \text {tr }}=\text { false, }\end{array}\right.$

$\dot{V}_{y}\left\{\begin{array}{l}\left|\dot{V}_{y}\right|>\dot{V}_{y_{\text {trans _th }}}\left(V_{x}\right) \longrightarrow F_{2_{-} \text {tr }}=\text { true, } \\ \left|\dot{V}_{y}\right| \leq \dot{V}_{y_{\text {trans _th min }}}\left(V_{x}\right) \longrightarrow F_{1_{-} \text {tr }}=\text { false, }\end{array}\right.$

$\dot{\delta}\left\{\begin{array}{l}|\dot{\delta}| * f\left(V_{x}\right)>\dot{\delta}_{\text {trans_th }}\left(V_{x}\right) \longrightarrow F_{3_{-} \text {tr }}=\text { true }, \\ |\dot{\delta}| * f\left(V_{x}\right) \leq \dot{\delta}_{\text {trans_th min }}\left(V_{x}\right) \longrightarrow F_{3_{-} \text {tr }}=\text { false, }\end{array}\right.$

$\delta\left\{\begin{array}{l}{[\delta]>\delta_{\text {trans_th }}\left(V_{x}\right) \longrightarrow F_{4-\text { tr }}=\text { true },} \\ |\delta| \leq \delta_{\text {trans_th min }}\left(V_{x}\right) \longrightarrow F_{4 \_ \text {tr }}=\text { false. }\end{array}\right.$

Transient mode

=delay_sample $\left\{\left(F_{1_{-} \text {tr }} \vee F_{2_{-} \text {tr }} \wedge F_{3_{-} \text {tr }} \wedge F_{4_{-} \text {tr }}\right), n\right\}$.
The delay mode starts when the input signal becomes false. The variable count is incremented by one as long as the old value of the variable is less than $n$, where $n$ is the number of samples. The output in this case is only true if the old value is less than $n$. Thus, the falling edge is delayed by $n$ samples. The reason for introducing the delay sample function is to avoid transient mode switching during signals' zero crossing.

We define three intermediate flags $F_{i_{-} \text {cr }}, i=1,3$. The steady turn is determined as follows:

$$
\begin{aligned}
& \dot{\psi}\left\{\begin{array}{l}
|\dot{\psi}|>\dot{\psi}_{\text {cr_th }} \longrightarrow F_{1_{-} \text {cr }}=\text { true } \\
|\dot{\psi}| \leq \dot{\psi}_{\text {cr_th min }} \longrightarrow F_{1_{-} \text {cr }}=\text { false }
\end{array}\right. \\
& \delta\left\{\begin{array}{l}
|\delta|>\delta_{\text {cr_th }} \longrightarrow F_{2_{-} \text {cr }}=\text { true }, \\
|\delta| \leq \delta_{\text {cr_th min }} \longrightarrow F_{2 \_ \text {cr }}=\text { false }
\end{array}\right. \\
& a_{y}\left\{\begin{array}{l}
\left|a_{y}\right|>a_{y_{\mathrm{cr}_{-} \mathrm{th}}} \longrightarrow F_{3_{-} \mathrm{cr}}=\text { true, } \\
\left|a_{y}\right| \leq a_{y_{\mathrm{cr}_{\mathrm{th}} \mathrm{min}}} \longrightarrow F_{3_{-} \mathrm{cr}}=\text { false, }
\end{array}\right.
\end{aligned}
$$

Steady turn $=\left\{F_{1_{-} \text {cr }} \vee F_{2_{-} \text {cr }} \wedge F_{3 \_c r}\right\}$.

Figure 3 illustrates the driving states algorithm described earlier. 
2.2.2. The Lateral Surface Capability Index. The lateral surface capability index is determined based on the comparison between actual vehicle motion obtained from sensor inputs and vehicle behavior obtained from a linear vehicle motion model. When the vehicle is in the linear range of operation, the vehicle motion model is close to the actual vehicle motion obtained from the sensor inputs. In this case, the lateral surface index is set to the maximum lateral acceleration that the vehicle can sustain on dry surface. When the vehicle lateral motion approaches the limit of adhesion, the vehicle motion model is substantially different from the actual vehicle motion, and it can be concluded that the lateral surface index must at least equal $a_{y} / g$, wherein $g$ denotes the gravity acceleration.

The first step in this block is to detect whether the vehicle is in a linear mode of motion or not. To this effect, the linear mode detection evaluates the following three conditions:

$$
\begin{aligned}
& \left|\dot{\psi}_{d} v_{x}+\dot{v}_{y d}\right|-\left|a_{y}\right|<a_{y_{\mathrm{Thr} 1}}, \\
& \left(\dot{\psi}_{d} v_{x}+\dot{v}_{y d}\right) a_{y}>-a_{y_{-} \mathrm{Thr} 2}, \\
& \dot{\psi}_{e_{-} \min } \leq\left|\dot{\psi}_{d}-\dot{\psi}\right| \leq \dot{\psi}_{e_{-} \max } .
\end{aligned}
$$

The desired yaw rate $\dot{\psi}_{d}$ is obtained from (23). $\dot{v}_{y d}$ is obtained by differentiating (18) as follows:

$$
\dot{v}_{y d}=\omega_{n}^{2}\left(x_{1}+\frac{1}{z_{v}}\left[V_{y \mathrm{dss}}-2 \xi \omega_{n} x_{1}-\omega_{n}^{2} x_{2}\right]\right) .
$$

The difference between measured and expected lateral accelerations in (28) is due to the fact that the assumed linear relationship between lateral force and sideslip angle does not hold exactly. In fact, the direct proportionality between lateral force and sideslip angle is a good approximation as long as the lateral forces are moderate. When the difference between measured and desired lateral accelerations exceeds a certain threshold, the sideslip angle increases much more strongly, indicating that the tire is moving towards its lateral limit of adhesion.

Equation (29) compares the product of signed desired and observed lateral accelerations, to a small negative number

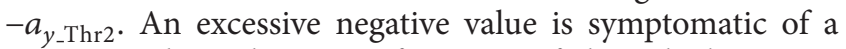
situation where the state of motion of the vehicle cannot follow the driver intended steering. Equation (30) compares the difference between a desired and observed yaw rate to upper and lower thresholds. Obviously, if this difference is above the upper threshold $\dot{\psi}_{e_{-} \max }$, control of the vehicle is not exact indicating that the vehicle has departed from its linear range of operation. On the other hand, if it is below the lower threshold $\dot{\psi}_{e_{-} \text {min }}$, it is likely that the vehicle is going straight, and that no information about the lateral friction properties of the road surface can be inferred from the data of the various sensors.
We define four intermediate flags $f_{i \operatorname{lin}} i=1,4$. The linear mode detection is determined as follows:

$$
\begin{aligned}
& \text { if }\left|\dot{\psi}_{d} v_{x}+\dot{v}_{y d}\right|-\left|a_{y}\right|<a_{y_{-} \text {Thr } 1} \rightarrow f_{1 \text { lin }}=\text { true, } \\
& \text { else } f_{1 \text { lin }}=\text { false, } \\
& \text { if }\left(\dot{\psi}_{d} v_{x}+\dot{v}_{y d}\right) a_{y}>-a_{y_{-} \text {Thr } 2} \longrightarrow f_{2 \text { lin }}=\text { true, } \\
& \text { else } f_{2 \text { lin }}=\text { false, } \\
& \text { if }\left|\dot{\psi}_{d}-\dot{\psi}\right| \leq \dot{\psi}_{e_{-} \text {max }} \longrightarrow f_{3 \text { lin }}=\text { true, } \\
& \text { else } f_{3 \text { lin }}=\text { false, } \\
& \text { if } \dot{\psi}_{e_{-} \text {min }} \leq\left|\dot{\psi}_{d}-\dot{\psi}\right| \rightarrow f_{4 \text { lin }}=\text { true, } \\
& \text { else } f_{4 \text { lin }}=\text { false. }
\end{aligned}
$$

The linear flag is given by

$$
f_{\text {lin }}(t)=f_{\text {lin }}(t-1) \vee f_{4 \operatorname{lin}} \wedge\left\{f_{1 \text { lin }} \wedge f_{2 \text { lin }} \wedge f_{3 \operatorname{lin}}\right\} .
$$

The second step in the lateral surface capability index block is the straight driving mode detection algorithm. We define the following intermediate flags $f_{\dot{\psi} \max }, f_{\dot{\psi} \min }, f_{\delta \max }$, and $f_{\delta \text { min }}$. The straight line flag is determined as follows:

$$
\begin{aligned}
& \text { if }|\dot{\psi}| \geq \dot{\Omega}_{\text {th max }} \longrightarrow f_{\dot{\text { max }}}=\text { false, } \\
& \text { else } f_{\psi \max }=\text { true, } \\
& \text { if }|\delta| \geq \delta_{\text {th } \max } \longrightarrow f_{\delta \text { max }}=\text { false, } \\
& \text { else } f_{\delta \text { max }}=\text { true, } \\
& \text { if }|\dot{\psi}| \leq \dot{\Omega}_{\text {th min }} \longrightarrow f_{\dot{\psi} \min }=\text { true, } \\
& \text { else } f_{\dot{\psi} \min }=\text { false, } \\
& \text { if }|\delta| \leq \delta_{\text {th } \min } \longrightarrow f_{\delta \text { min }}=\text { true } \\
& \text { else } f_{\delta \text { min }}=\text { false. }
\end{aligned}
$$

The straight driving flag is given by

$$
f_{\mathrm{sl}}(t)=f_{\mathrm{sl}}(t-1) \vee\left\{f_{\dot{\psi} \min } \wedge f_{\dot{\psi} \max } \wedge f_{\delta \min } \wedge f_{\delta \max }\right\} .
$$

The lateral surface capability index is determined based on input data from the sensors and the flags $f_{\mathrm{sl}}(t)$ and $f_{\text {lin }}(t)$. The operation is described referring to the flowchart of Figure 4. During the initialization step, the index $\mu$ is set to a predetermined default value $\mu_{0}$, which may be a typical friction coefficient of a dry, solid road surface. The lateral acceleration $a_{y}$ is read from lateral acceleration sensor. The straight line flag $f_{\mathrm{sl}}(t)$ is then verified. If it is "false," that is, if the vehicle is going through curves and is subject to a substantial lateral acceleration, a timer is reset to zero. Next, if the linear flag $f_{\operatorname{lin}}(t)$ is "true," it can be concluded that the lateral surface capability index must at least equal $a_{y} / g$, wherein $g$ denotes the gravity acceleration. The lateral surface capability index is therefore updated to be $\mu(t)=\max \left(\mu(t-1), a_{y}(t) / g\right)$ the maximum of $a_{y} / g$ and an estimate obtained from a previous iteration $\mu(t-1)$. In this way, if this step is executed repeatedly in subsequent iterations 


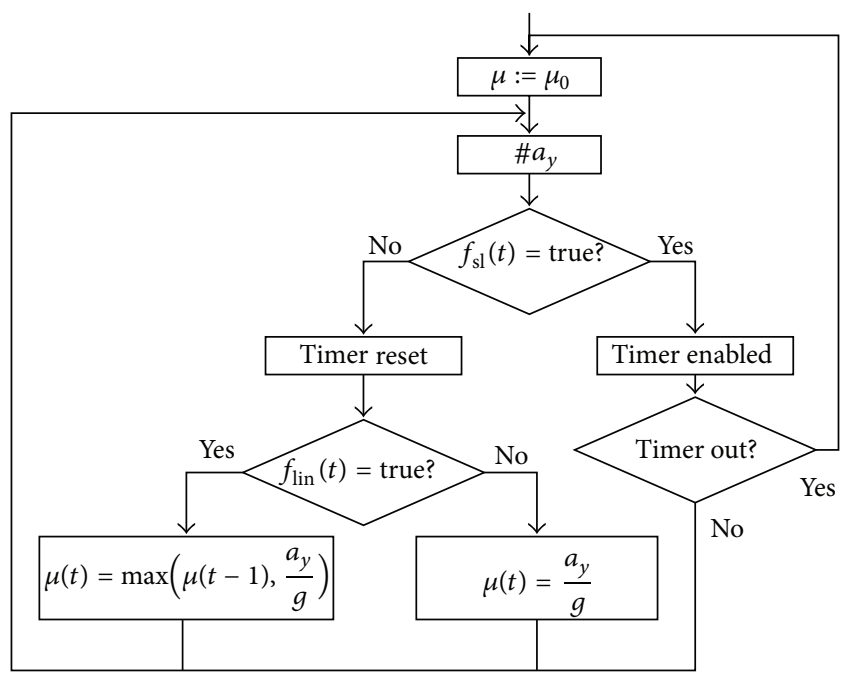

FIGURE 4: Schematic diagram of the lateral surface capability index.

of the procedure of Figure $4, \mu(t)$ will grow and converge towards the true friction coefficient of the road surface.

On the other hand, if the linear flag is found to be "false," this may be due to the fact that the quality of the road surface has deteriorated and its friction coefficient has decreased, or that the vehicle is going at the stability limit. In that case, $a_{y} / g$ is set as the new the lateral surface capability index.

If the straight driving flag is found to be "true," no estimation of lateral surface capability index is possible. In this case, the timer mentioned is enabled; that is, the timer starts to run if the straight flag has just switched to "true," or it simply continues to run if the straight flag was "true" already in the previous iteration of the procedure. The value of the timer is thus representative of the time in which the vehicle has been going straight. If the timer has exceeded a predetermined limit, the algorithm resets the friction coefficient to $\mu_{0}$. In this way, if the vehicle has been going straight for such a long time such that the previously acquired estimate of the lateral surface capability index is no longer reliable, the estimate is reset to $\mu_{0}$, and the process of iteratively approximating its true value restarts when the straight driving flag $f_{\text {sl }}(t)$ becomes "false."

2.2.3. The Warning Algorithm. The warning algorithm computes indices based on yaw-rate error, yaw rate dead-band, side slip velocity error, and side slip velocity dead-band, an understeer error, and an understeer dead-band, using various sensors. It consists of 3 major blocks:

(i) stability index block;

(ii) steady state linear/nonlinear understeer index block;

(iii) arbitration block.

Stability Index Block. The stability index block is mainly used during transient driving situations, in which a driver has to turn the steering wheel of the vehicle quickly and/or in alternating directions, so that a reliable estimation of

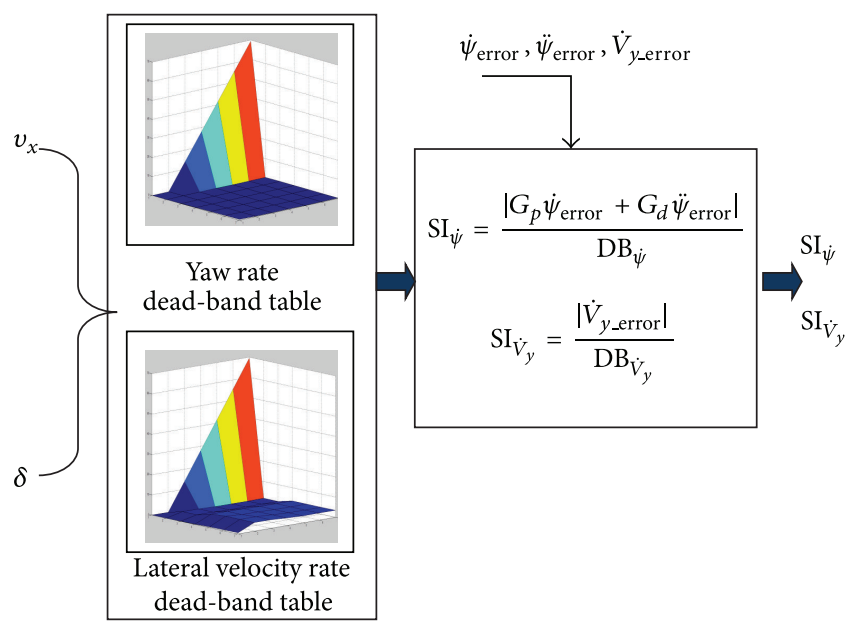

FIGURE 5: Schematic diagram of stability index implementation.

the vehicle understeer is difficult. Figure 5 illustrates the schematic diagram of the stability index implementation.

The stability index block requires the yaw rate error $\dot{\psi}$ error' the rate of the yaw error $\ddot{\psi}_{\text {error }}$, and the lateral velocity error rate $\dot{V}_{y \text { _error }}$ for the computation of the stability index. These quantities are defined as follows:

$$
\begin{gathered}
\dot{\psi}_{\text {error }}=\dot{\psi}_{d}-\dot{\psi}, \quad \ddot{\psi}_{\text {error }}=\frac{\left(\dot{\psi}_{\text {error }}(t)-\dot{\psi}_{\text {error }}(t-T)\right)}{T}, \\
\dot{V}_{y_{\text {error }}}=\dot{v}_{y d}-\dot{v}_{y}=\dot{v}_{y d}-\left(\dot{\psi} v_{x}-a_{y}\right) .
\end{gathered}
$$

The yaw rate and the lateral velocity rate dead-band lookup tables store a yaw rate dead-band $\mathrm{DB}_{\dot{\psi}}$ and a lateral velocity rate dead band $\mathrm{DB}_{\dot{V}_{y}}$, as a function of longitudinal velocity $v_{x}$ and steering wheel angle $\delta$. These dead bands represent (sometimes called a neutral zone) an area of a signal range or band where no action occurs (the system is dead), The purpose is of the dead band to prevent oscillation or repeated activation-deactivation cycles (called "hunting" in control systems). a predetermined percentage of a yaw rate or a lateral velocity derivative above which, for a given vehicle speed and steering wheel angle, control over the vehicle is lost. The warning algorithm is used in combination with an electronic stability control (ESC system), and the ESC system may use associated dead bands of the yaw rate and the lateral velocity derivative for deciding whether to intervene or not. If, for example, the dead bands of the ESC system are at $70 \%$ of a value at which control of the vehicle is lost for a given vehicle speed and steering wheel angle, the dead bands used in the stability index block may be at $50 \%$ of such a value, ensuring that the warning algorithm will issue a warning signal prior to any intervention of the ESC. 


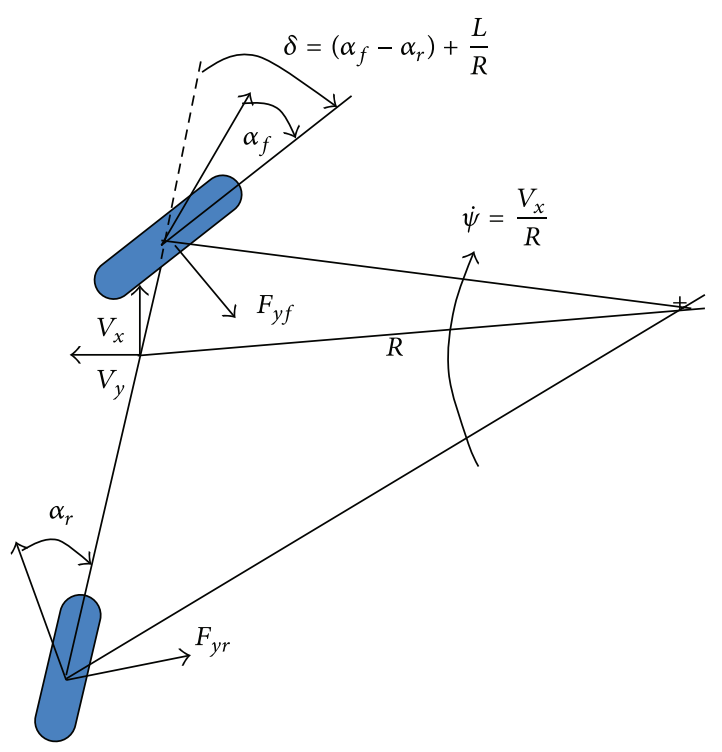

Figure 6: Vehicle in steady turn.

From the dead bands $\mathrm{DB}_{\dot{\psi}}, \mathrm{DB}_{\dot{V}_{y}}$ and the error signals $\dot{\psi}_{\text {error }}, \ddot{\psi}_{\text {error }}$, and $\dot{V}_{y \_ \text {error }}$, the yaw rate and lateral velocity rate stability indices $\mathrm{SI}_{\dot{\psi}}, \mathrm{SI}_{\dot{V}_{y}}$ as follows:

$$
\begin{gathered}
\mathrm{SI}_{\dot{\psi}}=\frac{\left|G_{p} \dot{\psi}_{\text {error }}+G_{d} \ddot{\psi}_{\text {error }}\right|}{\mathrm{DB}_{\dot{\psi}}}, \\
\mathrm{SI}_{\dot{V}_{y}}=\frac{\left|\dot{V}_{y_{\text {_error }}}\right|}{\mathrm{DB}_{\dot{V}_{y}}} .
\end{gathered}
$$

The decision whether the vehicle is in a situation approaching ESC activation or not during a transient maneuver can be based on the yaw rate and lateral velocity derivative indices defined in (37), and a general stability index SI is set equal to

$$
\mathrm{SI}=\max \left(\mathrm{SI}_{\dot{\psi}}, \mathrm{SI}_{\dot{i}_{y}}\right) .
$$

Steady State Linear/Nonlinear Understeer Index Block. In this section, we will discuss the structure and operation of the understeer index. For estimating the understeer of a vehicle, it is important to know whether the vehicle is moving in a linear regime, in which the sideslip angle of the vehicle is approximately directly proportional to the lateral forces. Figure 6 illustrates the vehicle in a steady turn; to keep the slip angle small, we assume that the turn radius is large. This is normally the case for high-speed turns, in which the vehicle is not skidding.

For steady state mode, the dynamic equation of motion in the body-centered coordinate system is given by

$$
\begin{aligned}
& \frac{M v_{x}^{2}}{R}=F_{y f}+F_{y r}, \\
& 0=a F_{y f}+b F_{y r} .
\end{aligned}
$$

This allows solving for the forces

$$
\begin{aligned}
& F_{y f}=\frac{a M v_{x}^{2}}{L R}, \\
& F_{y r}=\frac{b M v_{x}^{2}}{L R} .
\end{aligned}
$$

Using the relation $\dot{\psi}=v_{x} / R$, the steer angle equation can be written as

$$
\delta=-\alpha_{f}+\alpha_{r}+\frac{L}{R} .
$$

The linearized lateral forces are expressed in terms of the tire slip angles

$$
\begin{aligned}
& F_{y f}=C_{f} \alpha_{f}, \\
& F_{y r}=C_{r} \alpha_{r} .
\end{aligned}
$$

From (40) and (42), the steer angle relation becomes

$$
\delta=\left(\frac{M\left(b C_{r}-a C_{f}\right)}{L C_{f} C_{r}}\right) \frac{v_{x}^{2}}{R}+\frac{L}{R} .
$$

Equation (43) relates the steer angle to the speed via the understeer coefficient $K_{\text {und }}$

$$
\delta=K_{\mathrm{und}} \frac{v_{x}^{2}}{R}+\frac{L}{R},
$$

where

$$
\begin{aligned}
K_{\text {und }} & =\left(\frac{M\left(b C_{r}-a C_{f}\right)}{L C_{f} C_{r}}\right) \\
& =\frac{M}{L}\left(\frac{b}{C_{f}}-\frac{a}{C_{R}}\right) .
\end{aligned}
$$

From (44), we can calculate the change in the steer angle when the $L / R$ is changed by differentiating (44).

In the linear case

$$
\begin{gathered}
\frac{\partial \delta}{\partial(L / R)}=K_{\text {und_linear }} \frac{V_{x}^{2}}{L}+1, \\
K_{\text {und_linear }}=\frac{L}{V_{x}^{2}}\left(\frac{\partial \delta}{\partial(L / R)}-1\right) .
\end{gathered}
$$

Figure 7 shows the steering wheel angle $\delta$ versus $L / R$ for constant speed. For the linear tire force assumption, the slopes of the plot are constants since the understeer and the vehicle speed are constant.

For the nonlinear tire characteristics, in which the tire force-sideslip angle becomes significantly nonlinear, the slopes are not constant and vary with the turn radius when the vehicle speed is constant as illustrated in Figure 8. In this case, the understeer relates to the local slope of the steer angle curve. 


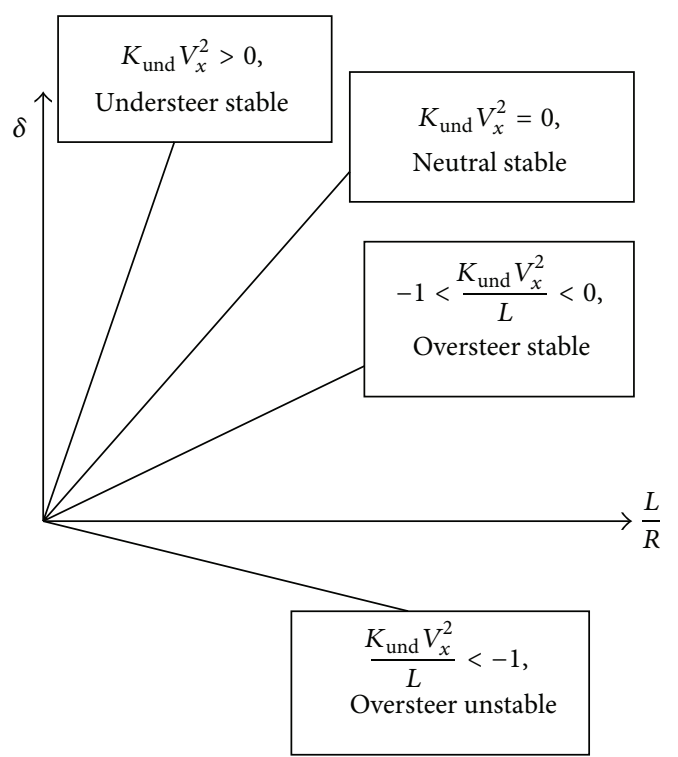

Figure 7: Steer angle versus $L / R$ for linear case [14].

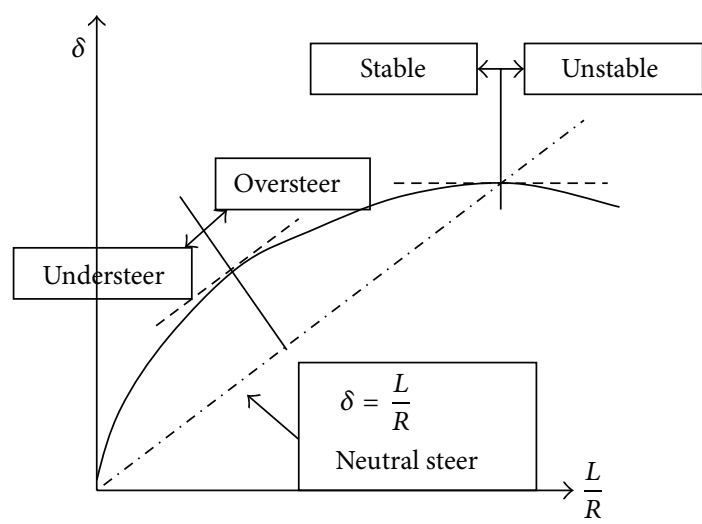

FIGURE 8: Steer angle versus $L / R$ for nonlinear case [14].

Since in the nonlinear case there is no constant understeer coefficient, we define a variable coefficient that expresses how the slip angle difference changes as the lateral acceleration changes

$$
K_{\text {und_nl }}=\frac{d\left(\alpha_{f}-\alpha_{r}\right)}{d\left(V_{x}^{2} / R g\right)}=\frac{d\left(\alpha_{f}-\alpha_{r}\right)}{d\left(a_{y}\right)} \text {, }
$$

where

$$
\left(\alpha_{f}-\alpha_{r}\right)=\delta-\frac{a+b}{v_{x}} \dot{\psi}=\delta-\frac{L a_{y}}{v_{x}^{2}} .
$$

By applying Kalman filter technique, it is possible to estimate the linear understeer and nonlinear understeer variable.

Estimation of the Linear and Nonlinear Understeer. In the last section, we developed linear and nonlinear expression for the understeer tendency of the vehicle under steady cornering.
In this section, an identification algorithm using the Kalman filter is developed to estimate the linear understeer and nonlinear understeer variable. The Kalman filter is a set of mathematical equations that provide an efficient computational (recursive) solution of the least-squares method. The filter is very powerful in several aspects; it supports estimations of past, present, and even future states, and it can do so even when the precise nature of the modeled system is unknown.

The Kalman filter addresses the general problem of trying to estimate the state of a discrete-time controlled process that is governed by the linear stochastic difference equation

$$
x(t+1)=x(t)+v(t)
$$

with a measurement $y \in \mathfrak{R}$ that is

$$
y(t)=H(t) x(t)+n(t) .
$$

We will be using two Kalman filters for the linear and nonlinear operations. Equations (44) and (47) can be recast as

$$
\begin{aligned}
& K_{\mathrm{und}} a_{y}=\delta-L \frac{a_{y}}{v_{x}^{2}}, \\
& K_{\mathrm{und}_{\mathrm{nl}}} \frac{d a_{y}}{d t}=\frac{d\left(\alpha_{f}-\alpha_{r}\right)}{d t} .
\end{aligned}
$$

In the linear range we define

$$
x(t)=K_{\text {und }}(t), \quad y(t)=\delta-L \frac{a_{y}}{v_{x}^{2}}, \quad H(t)=a_{y} .
$$

In the nonlinear range we define

$$
x(t)=K_{\text {und_nl }}(t), \quad y(t)=\frac{d\left(\alpha_{f}-\alpha_{r}\right)}{d t}, \quad H(t)=\frac{d a_{y}}{d t} .
$$

The random variables $v(t)$ and $n(t)$ represent the process and measurement noise, respectively.

They are assumed to be independent (of each other), white, and with normal probability distributions

$$
\begin{aligned}
& p(v) \sim N(0, Q), \\
& p(n) \sim N(0, \Gamma) .
\end{aligned}
$$

$Q$ is the process noise covariance and, $\Gamma$ is measurement noise covariance. $H(t)$ in the measurement equations (52)(53) relates the state to the measurement $y(t)$. In practice, it will change with each time step or measurement.

Consider the signal model of (49)-(54), and assume that the initial state and noise sequences are jointly Gaussian. Let $\widehat{x}(t+1)$ denote the conditional mean of $x(t+1)$ given 


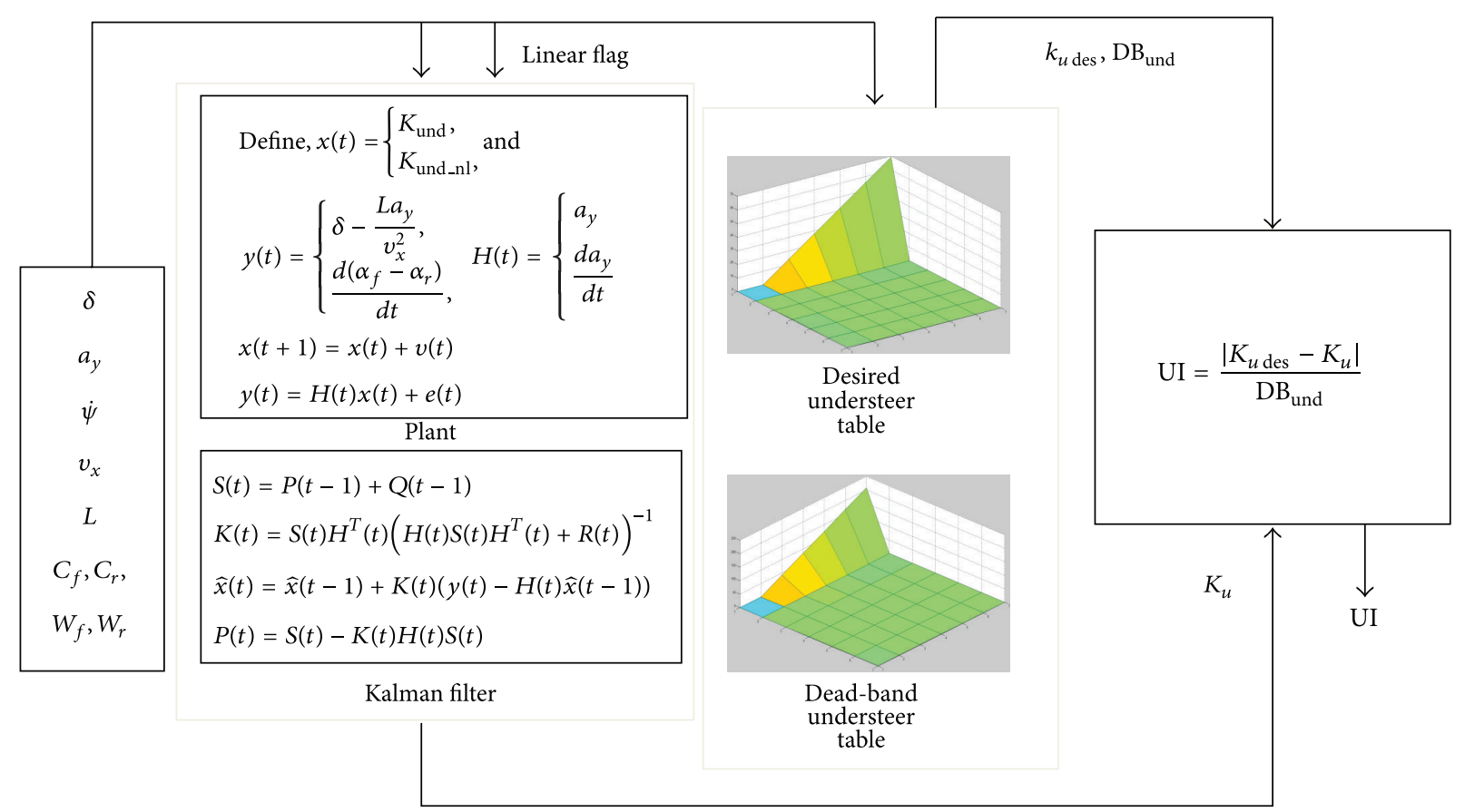

FIGURE 9: Schematic diagram of the understeer index implementation.

observation $\{y(t)\}$ up to and including time $t$; then, $\widehat{x}(t+1)$ satisfies the following recursion:

$$
\begin{gathered}
\widehat{x}(t+1)=\widehat{x}(t)+L(t)(y(t)-H(t) \hat{x}(t)), \\
\widehat{x}\left(t_{0}\right)=\bar{x}_{0}, \\
S(t+1)=P(t)+Q(t), \\
L(t)=S(t) H^{T}(t)\left(H(t) S(t) H^{T}(t)+\Gamma(t)\right)^{-1}, \\
P(t+1)=S(t+1)-L(t) H(t) S(t+1), \\
P\left(t_{0}\right)=\bar{P}_{0},
\end{gathered}
$$

where $L(t)$ is the filter gain and $P(t)$ is the state error covariance.

The linear flag defined in (35) controls the operation of the two Kalman filters (52) and (53) for estimating the understeer of the vehicle in linear and nonlinear regimes, respectively.

When the vehicle is determined to be in non-linear regime, the linear flag becomes zero, filter (52) stops, and filter (53) starts to operate, initialized with the most recent understeer value from filter (52). Similarly, when the linear flag changes back to 1, filter (52) becomes operative again and is initialized with a nominal understeer based on the front and rear lateral tire stiffness and the front and rear vehicle weight distribution and with initial covariance values.

In principle, the two filters might be regarded as a single Kalman filter which swaps $y(t)$ and $H(t)$ according to the value of the linear flag.

In analogy to what was described earlier for the yaw rate and lateral velocity rate stability indices $\mathrm{SI}_{\dot{\psi}}, \mathrm{SI}_{\dot{V}_{y}}$, lookup tables provided values of a desired understeer $K_{u \text { des }}$ and a dead band of the understeer $\mathrm{DB}_{\text {und }}$. As in case of the yaw rate and the lateral velocity derivative, desired understeer values can be predetermined empirically by measuring the understeer of a test vehicle at given speeds and steering wheel angles. Alternatively, they may be calculated in advance or in real time, for example, using the following formula:

$$
K_{u \text { des }}=\max \left(\frac{1}{v_{x}^{2}}\left(\frac{v_{x} \delta}{\dot{\psi}_{\text {des }}}-L\right), \frac{W_{f}}{C_{f}}-\frac{W_{r}}{C_{r}}\right) .
$$

Since $K_{u \text { des }}$ may take impractically high values according to (56), at high speeds and steering wheel angles, it is preferred to define an upper limit of the desired understeer $K_{u \text { des }}$ as follows:

$$
\begin{aligned}
& K_{u \text { des }} \\
& \quad=\min \left(\max \left(\frac{1}{v_{x}^{2}}\left(\frac{v_{x} \delta}{\dot{\psi}_{\text {des }}}-L\right), \frac{W_{f}}{C_{f}}-\frac{W_{r}}{C_{r}}\right), K_{\text {und_max }}\right) .
\end{aligned}
$$

The upper limit $K_{\text {und_max }}$ may be set to, for example, $8^{\circ} / g$ or $5^{\circ} / g, g$ denoting the gravity acceleration.

Similar to the yaw rate dead band $\mathrm{DB}_{\dot{\psi}}$ and a lateral velocity rate dead band $\mathrm{DB}_{\dot{V}_{y}}$, the understeer dead band $\mathrm{DB}_{\text {und }}$ gives values of the understeer which can be regarded as safe as function of longitudinal velocity $v_{x}$ and steering wheel angle $\delta$. The understeer index UI based on the effective vehicle understeer $K_{\text {und }}$ or $K_{\text {und_nl }}$, defined as $K_{u}$, estimated by filter (52) or (53) the desired understeer $K_{u \text { des }}$ from (57) and the understeer dead band $\mathrm{DB}_{\text {und }}$ :

$$
\mathrm{UI}=\frac{\left|K_{u \text { des }}-K_{u}\right|}{\mathrm{DB}_{\mathrm{und}}} .
$$

The structure and operation of the understeer index are depicted in Figure 9. 


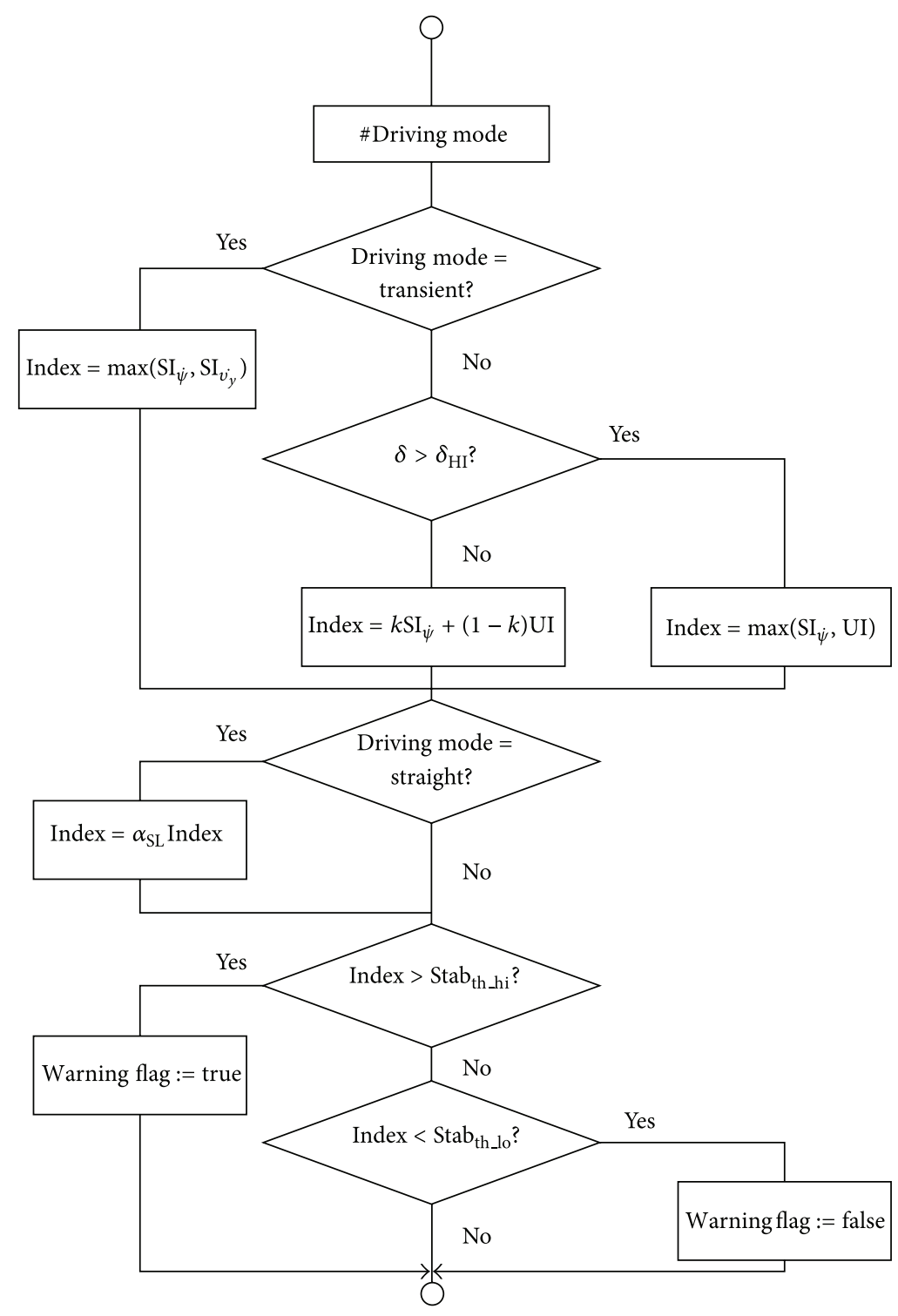

FIgURE 10: Flow chart of the arbitrator logic.

The Arbitrator Block. Referring again to Figures 5 and 9, three indices $\mathrm{SI}_{\dot{\psi}}, \mathrm{SI}_{i_{y}}$, and UI are supplied to the arbitrator. Albeit of different origin, the three indices are comparable in that they are dimensionless and that a value above 1 indicates a critical driving situation.

The operation of the arbitrator is explained referring to the flowchart of Figure 10. The first step is that we distinguish between a transient mode, and a steady state mode. In the transient mode the direction of the vehicle is changing so rapidly that the understeer cannot be relied upon. Therefore, if the driving mode is found to be the transient mode, the decision whether the vehicle is approaching ESC activation or not can only be based on the yaw rate and lateral velocity derivative indices $\mathrm{SI}_{\dot{\psi}}$ and $\mathrm{SI}_{\dot{v}_{y}}$.

A general stability index is set equal to the $\max \left(\mathrm{SI}_{\dot{\psi}}, \mathrm{SI}_{\dot{v}_{y}}\right)$. If the vehicle is not in the transient mode, the steering wheel angle $\delta$ is compared to a predetermined upper threshold $\delta_{\mathrm{HI}}$.
If this threshold is exceeded, there is a considerable risk of the vehicle being unstable, and the system should be rather liberal in issuing a warning. In that case, the general stability index is set equal to the $\max \left(\mathrm{SI}_{\dot{\psi}}, \mathrm{UI}\right)$. Otherwise, a weighted $\operatorname{sum}\left(k \mathrm{SI}_{\dot{\psi}}+(1-k) \mathrm{UI}\right)$ of the yaw rate stability index and understeer index is calculated. The weighting factor $k$ is tuned to a value between 0 and 1 . This weighting factor may be set dependent on the vehicle speed $v_{x}$ and decreases with the vehicle speed, giving increasing importance to the understeer at high speeds.

If the vehicle is in a straight line driving mode, the general stability index determined prior to this step is multiplied by positive factor $\alpha_{\mathrm{SL}}$, which is smaller than 1, reflecting the fact that the vehicle is least susceptible to a loss of control if it is driving a longer straight line.

Finally, the general stability index is compared to an upper threshold $\mathrm{Stab}_{\mathrm{th} \_\mathrm{hi}}$, for example, 0.8. If it exceeds this 
upper threshold, a warning flag is set to TRUE. Since the dead bands for warning algorithms are set lower than those of an ESC system, the warning flag will become TRUE prior to any intervention of the ESC system. If the stability index was found not to exceed the upper threshold, the index is compared to a lower threshold $\mathrm{Stab}_{\text {th_lo }}$, for example, 0.2. If it is below this lower threshold, the warning flag is set to FALSE; if not, the warning flag is left as it is until the procedure is repeated.

2.2.4. The Speed Advisory Block. Very often the road signs indicate the safe speed in a curve. However, on low $\mu$ surfaces, the suggested posted speed might not be adequate for the road condition.

When the vehicle is driving in a curve at a higher speed than the surface can allow, the understeer gradient of the vehicle increases causing the vehicle to plow or decreases and becomes negative causing the vehicle to spinout. The warning algorithm described earlier will issue a warning to alert the driver that he/she is traveling faster than the road surface can allow. In this section, we develop an advisory speed algorithm in a curve based on vehicle dynamics in conjunction with the driver warning algorithm. The advisory speed algorithm computes the advisory speed which allows a vehicle to travel around the turn or curve in its travel lane without causing an uncomfortable "side force" to its driver or passengers and helps maintain control of the vehicle. The advisory speed is based on the maximum lateral capability of the surface, the driver steering input, the actual vehicle speed, and the actual understeer of the vehicle. A visual advisory speed can be displayed, for example, in the DCI or a HUD display when the warning signal is issued. The visual advisory HMI is outside the scope of this paper.

Based on the steering input $\delta$ and vehicle speed $v_{x}$, if we assume that the coefficient of the surface would allow the vehicle (theoretically) to stay in the linear range, the desired understeer is determined from (57). If the surface coefficient is much lower, we can compute a vehicle speed (less than the actual speed of the vehicle) such that the understeer gradient is kept within a small deviation from the desired understeer of the vehicle. Assume that $\Delta K_{\text {und }}$ is the understeer deviation from the linear performance. Thus, the stable vehicle speed limit can be determined from (44) as follows:

$$
=\sqrt{\frac{v_{\lim }}{|\delta|-\left[\min \left(\max \left(\frac{1}{v_{x}^{2}}\left(\frac{v_{x} \delta}{\dot{\psi}_{\text {des }}}-L\right), \frac{W_{f}}{C_{f}}-\frac{W_{r}}{C_{r}}\right), K_{\text {und_max }}\right)+\Delta K_{\text {und }}\right] \mu g}},
$$

where $\mu$ is the maximum capability of the surface and is determined as described in Section 2.2.2.

Finally, the advisory speed is calculated as follows:

$V_{\mathrm{adv}}$

$=\left\{\begin{array}{l}\min \left(v_{\lim }, v_{x}\right) \text { if the warning flag is set } \\ \text { speed limit if available, when warning flag is not set. }\end{array}\right.$

Under straight line condition $v_{\lim }$ is set to $\infty$.

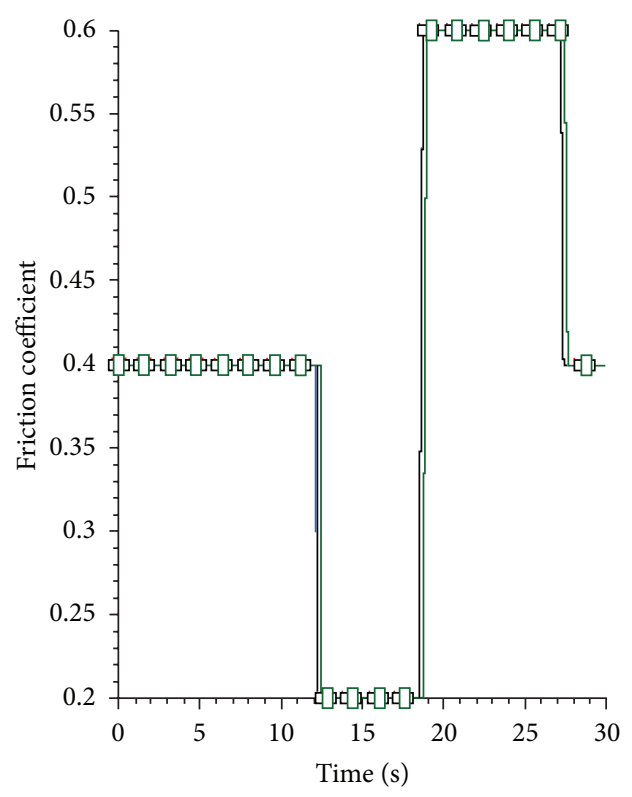

Figure 11: Time trace of the road friction coefficient.

The advisory speed is communicated to the driver to allow the driver to make a choice on what action should be taken, or through an intervention system where the engine and/or braking systems are controlled automatically to reduce the vehicle's speed.

\section{Simulation Results}

In this section, we present some typical simulation results showing the performance of the driver warning algorithm described in Section 2. The vehicle was traveling at $80 \mathrm{kph}$ on a $40 \mathrm{~m}$ radius loop with $200 \mathrm{~m}$ straight section with variable friction coefficient to simulate the exit ramp of a freeway. A driver model with a driver preview time of 1 second and driver lag of 0.12 second was used in the simulation. Figure 11 shows the time trace of the road friction coefficient as the vehicle travels. In this simulation, the driver did not react to the warning at the time when the warning was issued.

Figure 12 illustrates the time trace of the warning signal and the ESC activation flag. The warning was issued at around 5 seconds when the driver entered the curved section of the road at $80 \mathrm{kph}$. In order to make ESC system as unintrusive as possible, its activation threshold will have to be set rather high. If the vehicle starts to drift, the ESC system is activated to keep the vehicle back on course. Figure 13 shows the vehicle actual path as compared to the target path. It is noticed that although the ESC intervened to maintain the vehicle stability, the vehicle had started sliding towards the edge of the road and did not follow closely the target path. This is also confirmed in Figure 14, where the vehicle trajectory is illustrated as the vehicle entered the curved section of the road.

To illustrate the performance of the speed advisory algorithm, the simulation was repeated this time with the 


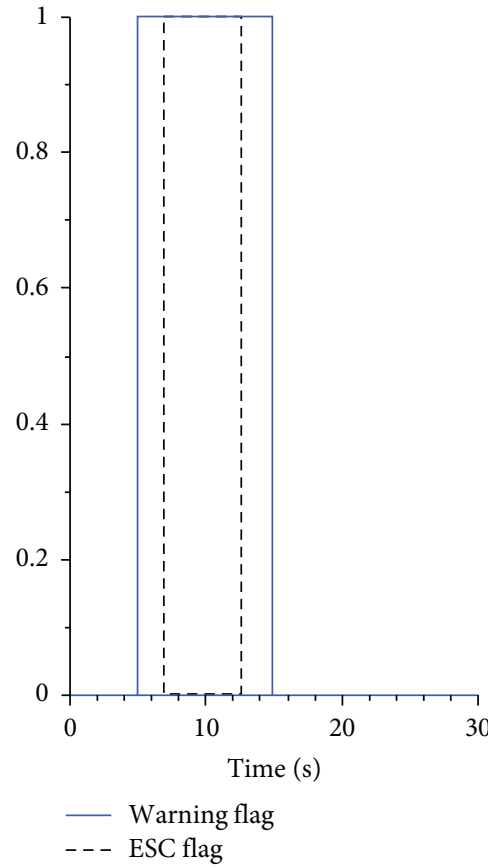

FIGURE 12: Warning and ESC flags time trace, driver not reacting to the warning at the time the warning is set.

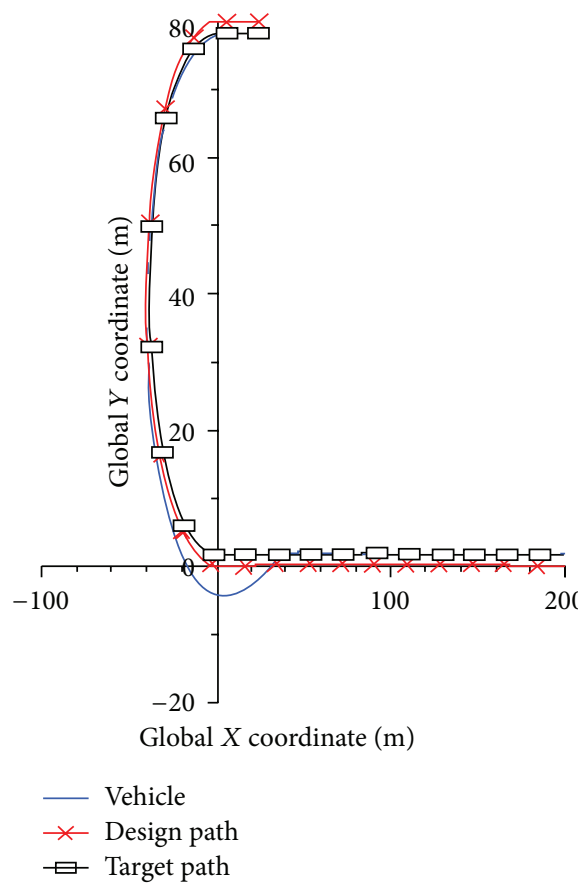

FIGURE 13: Vehicle path compared to the target path, driver not reacting to the warning at the time the warning is set.

driver reacting to the warning signal by reducing the vehicle speed based on the speed advisory algorithm.

Figure 15 shows the vehicle advisory speed and the vehicle speed as function of time. The advisory speed is initially set equal to the speed of the vehicle before the activation of the warning signal. This speed will not be displayed until

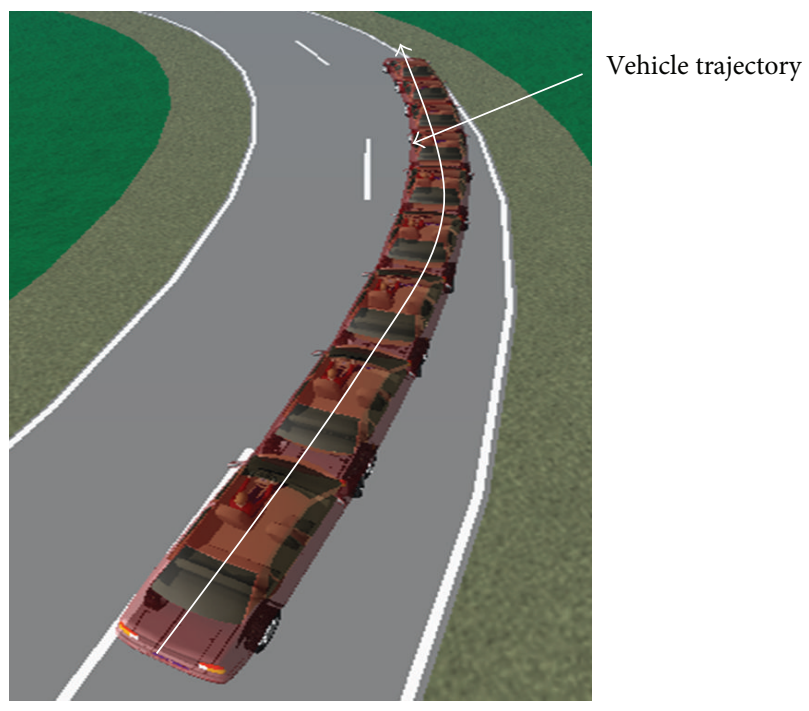

FIGURE 14: Vehicle trajectory in the curved section of the road, driver not reacting to the warning at the time the warning is set.

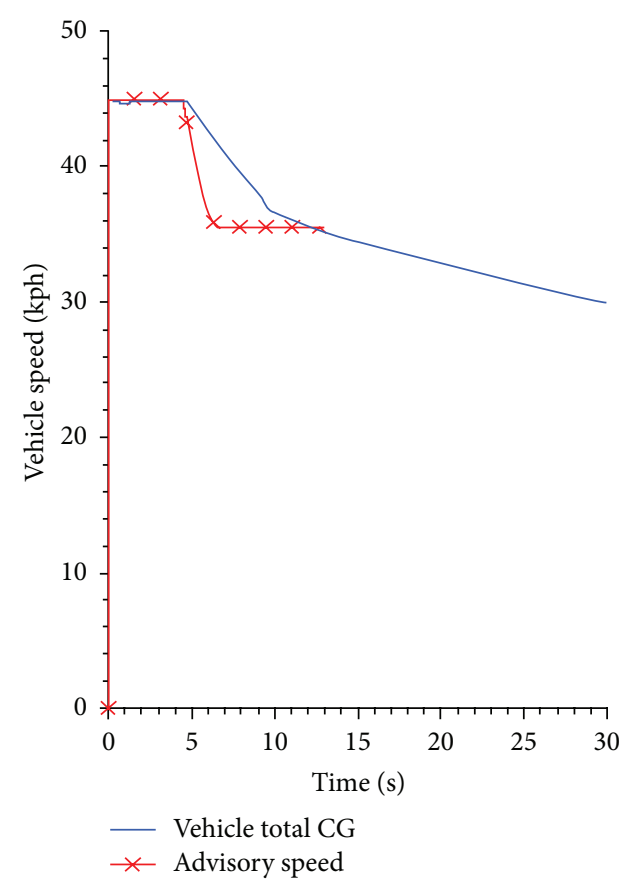

Figure 15: Vehicle advisory speed.

the warning is activated. At the end of the warning signal, the advisory speed will no longer be displayed to the driver. Instead if the vehicle is equipped with GPS receiver and a conventional navigation system, the GPS receiver enables the speed limit detection unit to find out the exact geographic location of the vehicle, to identify, based on map data of the navigation system, a road on which the vehicle is currently moving and to retrieve from the navigation system data on an eventual speed limit on this road. Figure 16 shows the vehicle trajectory; in this case, the vehicle is following more precisely the target path as opposed to the first case where the driver 


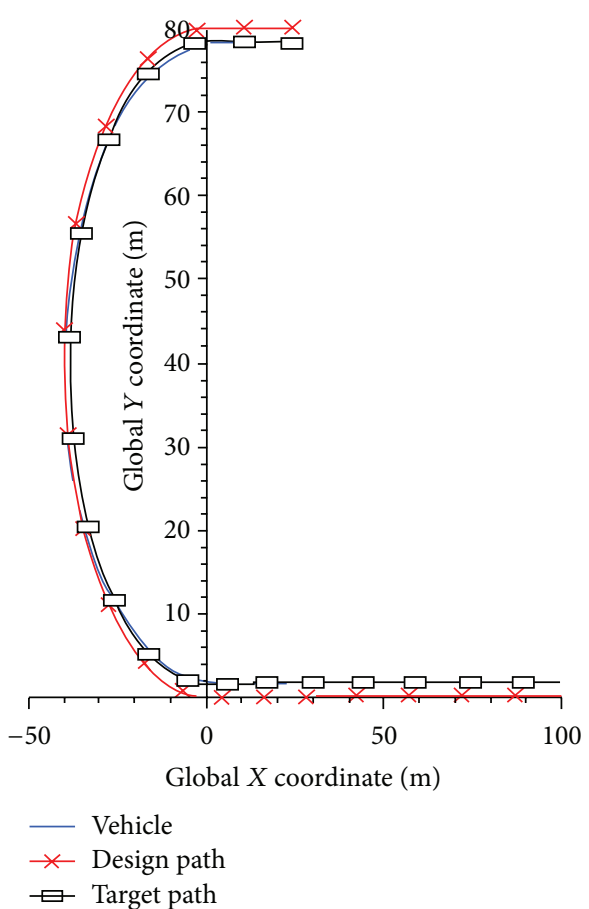

FIGURE 16: Vehicle path compared to the target path, driver reacting to the warning at the time the warning is set.

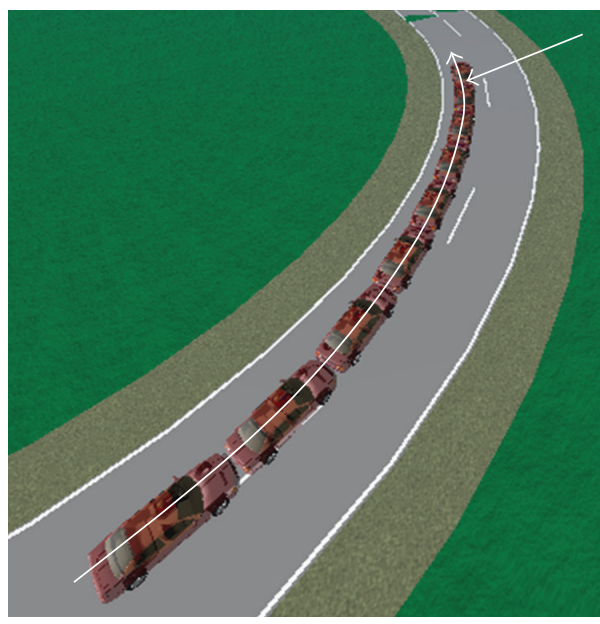

Vehicle trajectory

FIGURE 17: Vehicle trajectory in the curved section of the road, driver reacting to the warning at the time the warning is set.

did not react at the time the warning was set. Figure 17 shows a stable trajectory of the vehicle. It is also noticed that the duration of the warning signal has been reduced, and the ESC did not activate as shown in Figure 18.

To study the effect of the vehicle being on a banked road on the warning algorithm, the previous simulation was repeated with the vehicle traveling at $80 \mathrm{kph}$ on a $40 \mathrm{~m}$ radius loop with $200 \mathrm{~m}$ straight section of dry surface and variable bank angle. When the vehicle operator is driving on a banked road, the measured lateral acceleration will include the effect of the banked road and therefore affecting the straight driving

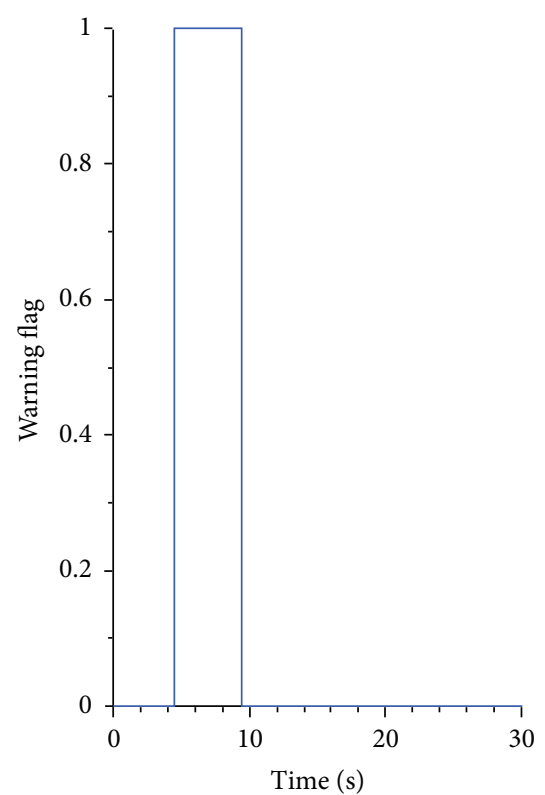

FIGURE 18: Warning and ESC flags time trace, driver reacting to the warning at the time the warning is set.

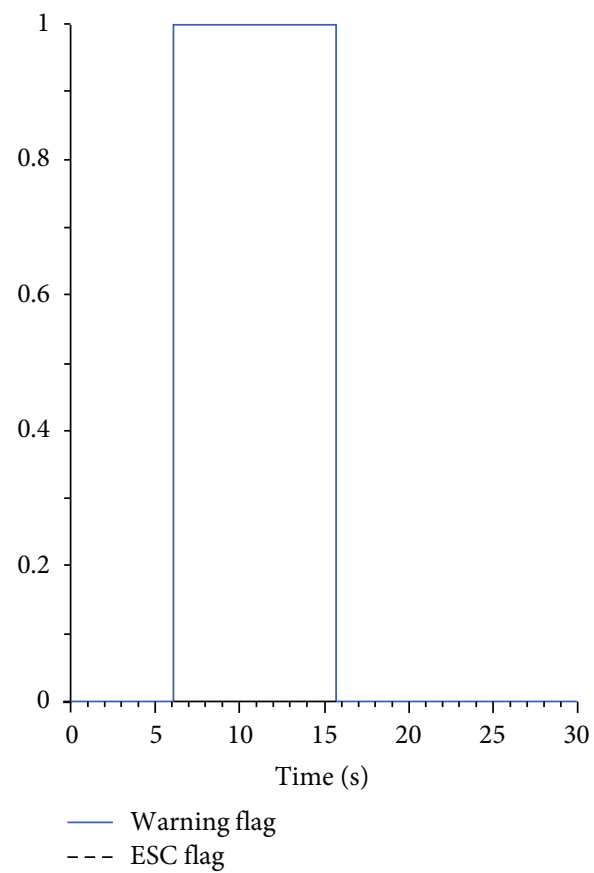

FIgURE 19: False warning issued on a banked road with uncompensated lateral acceleration.

detection algorithm. In addition, the operator introduces a correction to the steering angle to maintain the vehicle on the road, and therefore the desired yaw rate and understeer commands will indicate that the driver wishes to travel on the bank and not across it. In this case, the warning signal might be triggered unnecessarily especially when the vehicle is in the linear range of operation. Figure 19 shows that algorithm issued a warning signal even though the vehicle was stable 


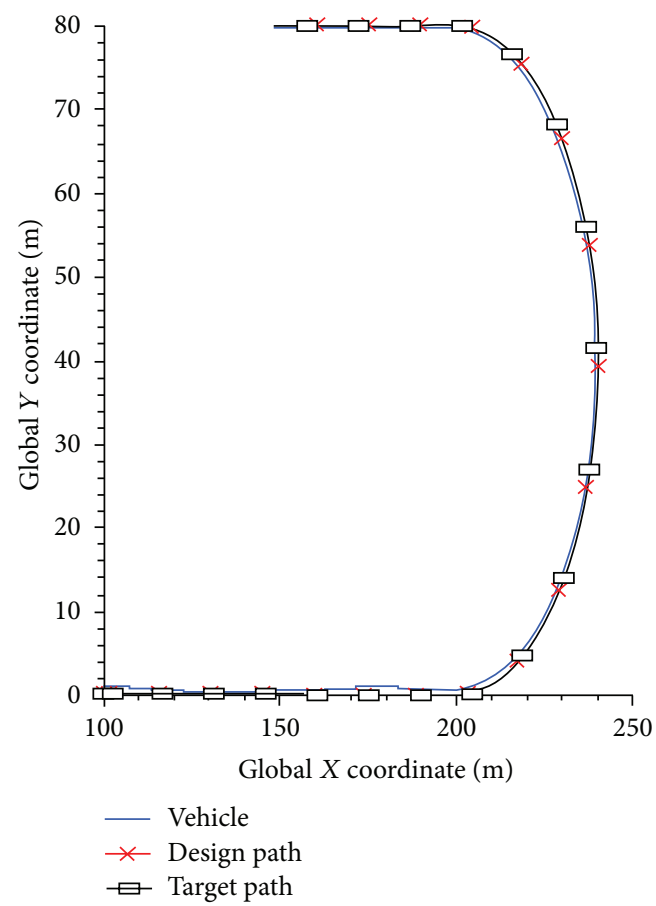

FIGURE 20: Vehicle path compared to the target path on a banked road.

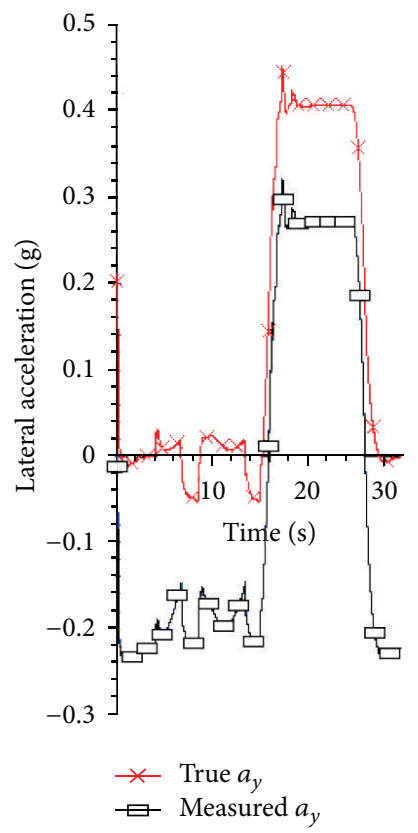

FIGURE 21: True and measured uncompensated lateral acceleration on a banked road.

and followed the target path as shown in Figure 20. Figure 21 compares the measured acceleration including the effect of the bank component to the true lateral acceleration of the vehicle.

Therefore, it is important to compensate for the effect of the bank in the lateral acceleration measurement.
When the vehicle is driven on a banked road, the measured lateral acceleration is corrupted by the bank angle of the road given by the following equation:

$$
a_{y m}=a_{y}+g \sin \phi
$$

The kinematics relationship between the lateral acceleration $a_{y}$ and the yaw rate of the vehicle $\dot{\psi}$ is given by the following equation:

$$
\dot{v}_{y}=a_{y}-\dot{\psi} v_{x}
$$

Under steady state equation, $\dot{v}_{y}=0$, and therefore (62) becomes

$$
a_{y}=\dot{\psi} v_{x}
$$

Define $\varepsilon(k)$ as

$$
\begin{aligned}
\varepsilon(k) & =a_{y m}(k)-a_{y}(k)=g \sin \phi \\
& =a_{y m}(k)-\dot{\psi} v_{x}(k) .
\end{aligned}
$$

Next, we will develop a Kalman filter to estimate $\varepsilon(k)$.

Define the following state vector

$$
x(k)=\left[\begin{array}{c}
1 \\
\varepsilon(k)
\end{array}\right]
$$

the state vector is governed by the linear stochastic difference equation

$$
x(k+1)=x(k)+\omega(k)
$$

with a measurement $y \in \Re$ that is

$$
y(k)=\dot{\varphi} v_{x}(k)=\left\lfloor a_{y m}(k)-1\right\rfloor x(k)+\eta(k) .
$$

The random variables $\omega(k)$ and $\eta(k)$ represent the process and measurement noise, respectively.

They are assumed to be independent (of each other), white, and with normal probability distributions

$$
\begin{aligned}
& p(\omega) \sim N(0, Q), \\
& p(\eta) \sim N(0, \Gamma) .
\end{aligned}
$$

The Kalman filter developed in (55) is applied to obtain an estimate $\widehat{\varepsilon}(k)$ for the difference between the measured and the actual lateral acceleration.

The previous simulation was repeated using the compensated lateral acceleration

$$
a_{y \text { comp }}=a_{y}+\widehat{\varepsilon}(k) .
$$

In Figure 22, the compensated measured lateral acceleration shows a good agreement with the true lateral acceleration of the vehicle. Referring to Figure 23, the simulation result represents an example of a correct warning (no warning issued) on a banked curve in the linear range of the vehicle when the lateral acceleration is compensated. 


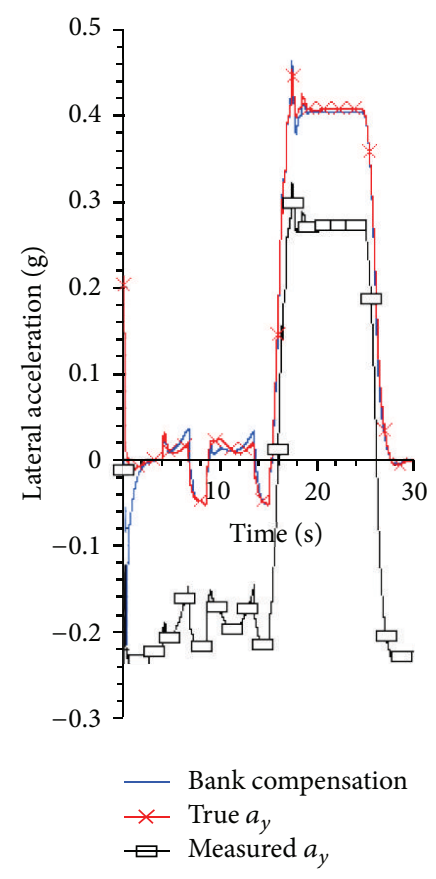

FIGURE 22: True lateral acceleration and measured uncompensated and compensated lateral acceleration on a banked road.

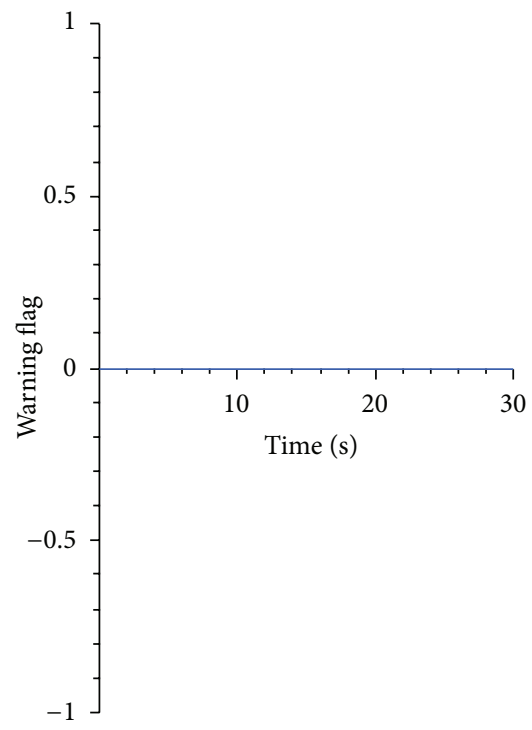

FIGURE 23: Warning signal on a banked road with compensated lateral acceleration.

\section{Vehicle Test Results}

To evaluate the warning algorithm performance, the following results are based on experimental data obtained using an Opel Omega vehicle equipped with ESC sensors. The tests were conducted on low-mu handling track with straight

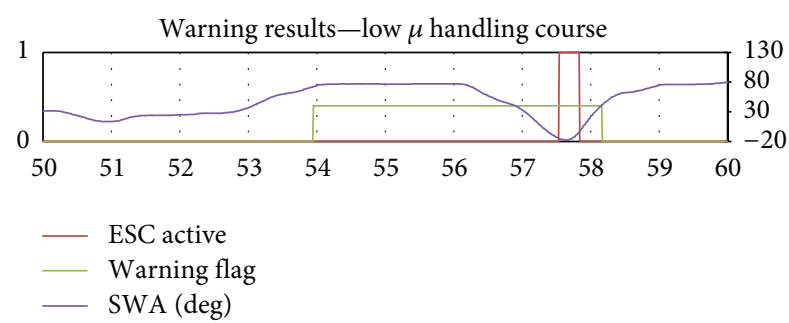

(a)

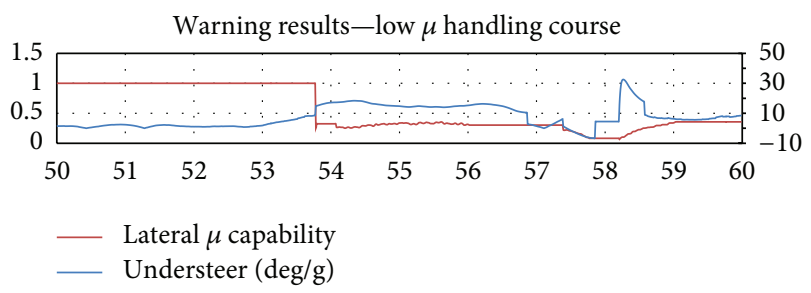

(b)

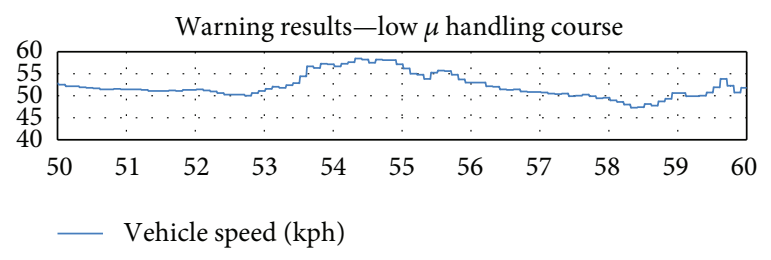

(c)

Figure 24: Vehicle simulating a freeway exit on low $\mu$ surface.

and curved sections to simulate a freeway exit. Time slices from different driving sessions are zoomed in to illustrate the performance of the warning algorithm.

A time slice of 10 seconds (50-60 seconds) is shown in Figure 24. The graphs show the performance of the warning system as the vehicle simulates a free way exit. In the first 4 seconds, the driver enters a curved section of the course.

At time $t=54$ seconds, the driver maintains an $80 \mathrm{deg}$ steering wheel angle at speed of $58 \mathrm{kph}$. The vehicle starts to understeer and reaches an understeer of $20 \mathrm{deg} / \mathrm{g}$. The lateral mu capability algorithm identifies a maximum lateral surface capability of 0.3 . At this moment, the warning signal was issued, and the driver released his accelerator pedal but did not apply his brakes. The ESC was activated 3.5 seconds after the warning signal and slowed down the car. The sudden change in the understeer at 58 seconds is due to the fact that vehicle was not in a steady state mode and the understeer is not defined outside the steady state behavior of the vehicle.

Figure 25 represents a series of transient maneuvers on the handling course. As seen from the warning signal and the ESC active flag, the warning signal was issued 3 to 4 seconds prior to the ESC activation which allows the driver to react to the warning signal and reduce the vehicle speed.

Figure 26 shows a large increasing steering angle maneuver at relatively low speed. It is noticed that under this scenario the warming is issued almost at the same time as the ESC activation. The warning signal is not effective in this case 


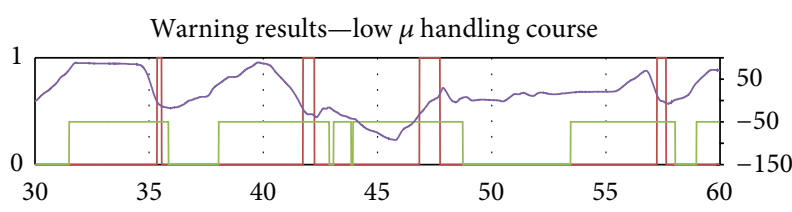

- ESC active

- Warning flag

- SWA (deg)

Figure 25: Warning and ESC signals under transient maneuvers.

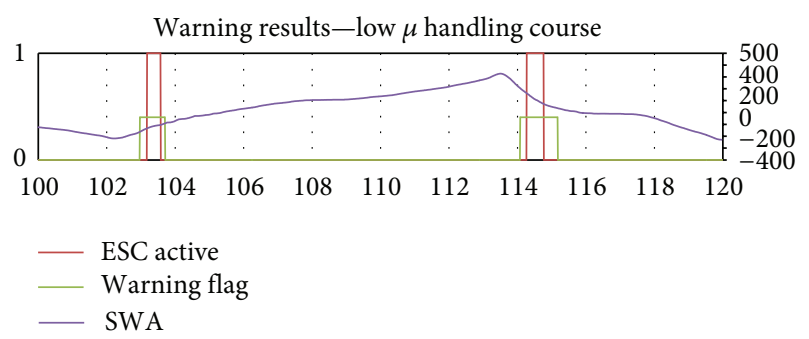

FIGURE 26: Warning and ESC signals with increasing steering angle at low speed.

since the driver does not have time to react to the warning signal before the ESC activation.

Figure 27 shows the vehicle going into a steady state maneuver. In this case, the speed advisory algorithm was triggered as the driver enters the curved section of the road. The vehicle starts to understeer and the warning signal is set. The driver releases the accelerator pedal and reduces the steering input. The warning signal was then terminated and the speed advisory ended. The ESC did not activate in this case since the driver reduced his steering input and the understeer gradient of the vehicle is reduced. The vehicle did not a have an automatic brake control to control the vehicle speed to the advisory speed. The warning was set until the driver reduced the steering input.

\section{Conclusions}

(1) In some of the conditions evaluated, the warning signal is issued 3 to 4 seconds prior to ESC activation to allow the driver to react to the warning.

(2) If the driver reacts to the warning signal by reducing the vehicle speed in the curve, the vehicle will follow more precisely the road curves with minimum or no ESC intervention.

(3) For large and increasing steering angle maneuver at relatively low speed, the warning is issued almost at the same time as the ESC activation. The warning signal is not effective in this case since the driver does not have time to react to the warning signal before the ESC activation. However, at these low speeds, the ESC is very effective and can stabilize the vehicle without heavy brake intervention.

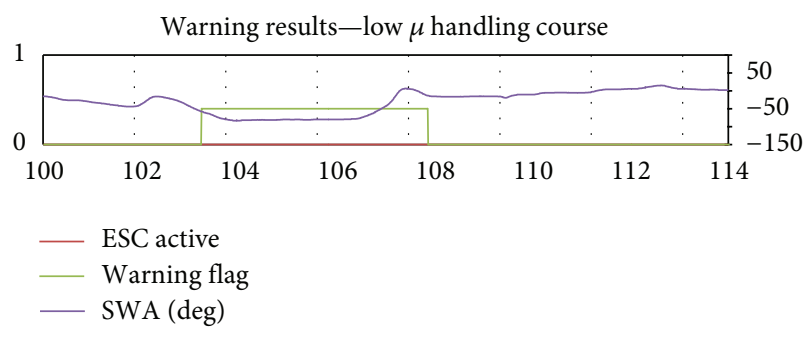

(a)

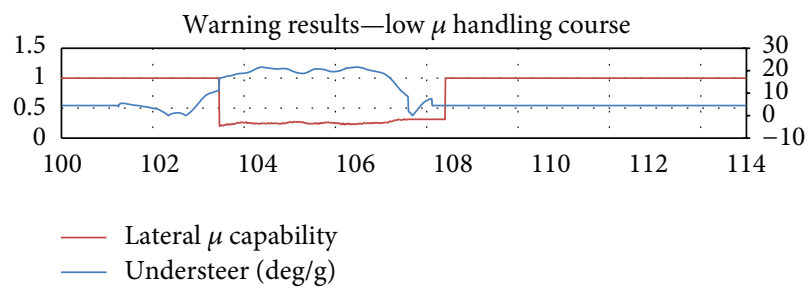

(b)

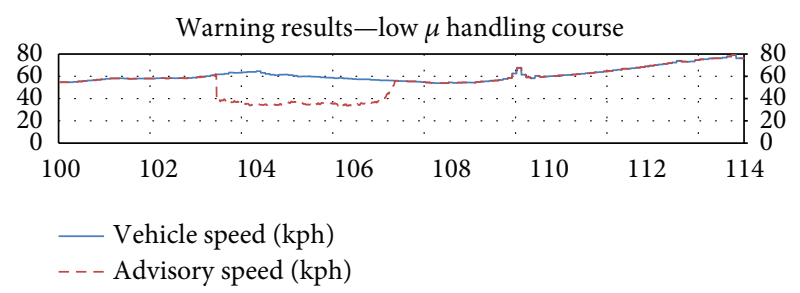

(c)

FIgURE 27: Vehicle simulating a freeway exit on low $\mu$ surface with speed advisory.

(4) When the vehicle is driven on a banked road, the uncompensated lateral acceleration measurement can false trigger the warning algorithm. Therefore, it is important to compensate for the effect of the bank in the lateral acceleration measurement.

(5) This warning system should be evaluated in a wider range of road and vehicle conditions to more fully evaluate its usefulness.

\section{Nomenclature}

$a$ : Distance from the center of gravity of vehicle to the front axle $(\mathrm{m})$

$b$ : Distance from the center of gravity of vehicle to the rear axle (m)

$C_{f}$ : Cornering stiffness of both tires of front axle $(\mathrm{N} / \mathrm{rad})$

$C_{r}$ : Cornering stiffness of both tires of rear axle (N/rad)

$g:$ Acceleration of gravity $\left(\mathrm{m} / \mathrm{s}^{2}\right)$

$g_{r}:$ Steering gear ratio

$I_{z}$ : Moment of inertia of entire vehicle about the yaw axis $\left(\mathrm{kgm}^{2}\right)$

$M_{v}$ : Total vehicle mass $(\mathrm{kg})$

$W_{f}$ : Vehicle weight on the front axle 


\begin{tabular}{|c|c|}
\hline$W_{r}:$ & Vehicle weight on the rear axle \\
\hline$K_{u}:$ & Desired understeer coefficient (deg/g) \\
\hline$T_{w}:$ & Vehicle track width $(\mathrm{m})$ \\
\hline$v_{y}:$ & $\begin{array}{l}\text { Lateral velocity of vehicle's center of } \\
\text { gravity }(\mathrm{m} / \mathrm{s})\end{array}$ \\
\hline$a_{y}:$ & $\begin{array}{l}\text { Lateral acceleration of vehicle's center of } \\
\text { gravity }\left(\mathrm{m} / \mathrm{s}^{2}\right)\end{array}$ \\
\hline$a_{y m}:$ & $\begin{array}{l}\text { Measured lateral acceleration at the } \\
\text { vehicle's center of gravity }\left(\mathrm{m} / \mathrm{s}^{2}\right)\end{array}$ \\
\hline$v_{y d}:$ & $\begin{array}{l}\text { Desired lateral velocity of vehicle's } \\
\text { center of gravity }(\mathrm{m} / \mathrm{s})\end{array}$ \\
\hline$\dot{v}_{y d}:$ & $\begin{array}{l}\text { Desired lateral velocity rate of vehicle's } \\
\text { center of gravity }\left(\mathrm{m} / \mathrm{s}^{2}\right)\end{array}$ \\
\hline$V_{y \text { gain }}:$ & Lateral velocity gain $(\mathrm{m} / \mathrm{s} / \mathrm{rad})$ \\
\hline$V_{y \mathrm{dss}}:$ & $\begin{array}{l}\text { Steady sate desired lateral velocity of } \\
\text { vehicle's center of gravity }(\mathrm{m} / \mathrm{s})\end{array}$ \\
\hline$v_{x}:$ & $\begin{array}{l}\text { Longitudinal velocity of vehicle's center } \\
\text { of gravity }(\mathrm{m} / \mathrm{s})\end{array}$ \\
\hline$x_{i}, i=1,2$ : & State variables of the second-order filter \\
\hline$z_{v}:$ & $\begin{array}{l}\text { Negative of system zero for desired side } \\
\text { slip velocity }\end{array}$ \\
\hline$z_{\dot{\psi}}:$ & $\begin{array}{l}\text { Negative of system zero for desired yaw } \\
\text { rate }\end{array}$ \\
\hline$\delta:$ & Steering angle of the front wheels (rad) \\
\hline$\dot{\psi}:$ & Yaw rate of vehicle $(\mathrm{rad} / \mathrm{s})$ \\
\hline$\Phi:$ & Bank angle (rad) \\
\hline$\dot{\psi}_{d}:$ & Desired yaw rate of vehicle (rad/s) \\
\hline$\dot{\psi}_{\mathrm{dss}}:$ & $\begin{array}{l}\text { Desired steady state yaw rate of vehicle } \\
(\mathrm{rad} / \mathrm{s})\end{array}$ \\
\hline$\Omega_{\text {gain }}:$ & Yaw velocity gain $(\mathrm{rad} / \mathrm{s} / \mathrm{rad})$ \\
\hline$\zeta:$ & $\begin{array}{l}\text { Damping ratio of desired vehicle } \\
\text { performance }\end{array}$ \\
\hline$\omega_{n}:$ & $\begin{array}{l}\text { Natural frequency of desired vehicle } \\
\text { performance }(\mathrm{rad} / \mathrm{s}) \text {. }\end{array}$ \\
\hline
\end{tabular}

\section{References}

[1] D. Hoffman and M. Rizzo, "Chevrolet C5 corvette vehicle dynamic control system," SAE Paper 980233, 1998.

[2] K. Jost, "Cadillac stability enhancement," Automotive Engineering, 1996.

[3] H. Nakazato, K. Iwata, and Y. Yoshiyoka, "A new system for independently controlling braking force between inner and outer rear wheels," SAE Paper 890835, 1989.

[4] H. Inagaki, K. Akuzawa, and M. Sato, "Yaw rate feedback braking force distribution control with control by wire braking system," in Proceedings of the International Symposium on Advanced Vehicle Control (AVEC '92), pp. 435-440, 1992.

[5] M. Salman, Z. Zang, and N. Boustany, "Coordinated control of four wheel braking and rear steering," in Proceedings of the American Control Conference, pp. 6-10, June 1992.

[6] T. Pilutti, G. Ulsoy, and D. Hrovat, "Vehicle steering intervention through differential braking," in Proceedings of the American Control Conference, June 1995.

[7] Y. -Shibahata, M. Abe, K. Shimada, and Y. Furukawa, "Improvement on limit performance of vehicle motion by chassis control," in Proceedings of the 13th Symposium on the Dynamics of Vehicles on Roads and Tracks (IAVSD '95), 1995.
[8] C. A. Sawyer, "Controlling vehicle stability," Automotive Industries, 1995.

[9] Y. A. Ghoneim, W. C. Lin, D. M. Sidlosky, H. H. Chen, Y. K. Chin, and M. J. Tedrake, "Integrated chassis control system to enhance vehicle stability," International Journal of Vehicle Design, vol. 23, no. 1, pp. 124-144, 2000.

[10] H. Leffler, R. Auffhammer, R. Heyken, and H. Roth, "New driving stability control system with reduced technical effort for compact and medium class passenger cars," SAE Paper 980234, 1998.

[11] J. Ackermann and S. Turk, "A common controller for a family of plant models," in Proceedings of the of the 21st IEEE Conference on Decision and Control (CDC '82), Orlando, Fla, USA, 1982.

[12] T. Pilutti, G. Ulsoy, and D. Hrovat, "Vehicle steering intervention through differential braking," in Proceedings of the American Control Conference. Part 1 (of 6), pp. 1667-1671, June 1995.

[13] G. Thomas, "Fundamentals of vehicle dynamics," Society of Automotive Engineers, Inc. Second Printing, 1992.

[14] D. karnopp, Vehicle Stability (Marcel Dekker), 2004. 

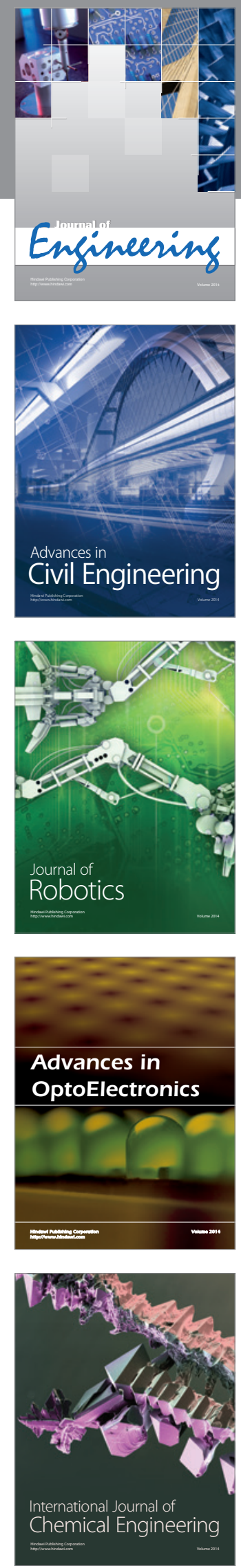

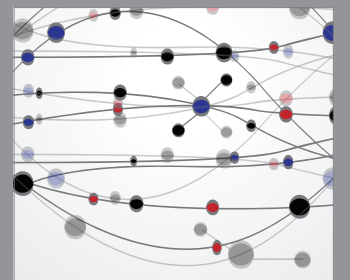

The Scientific World Journal
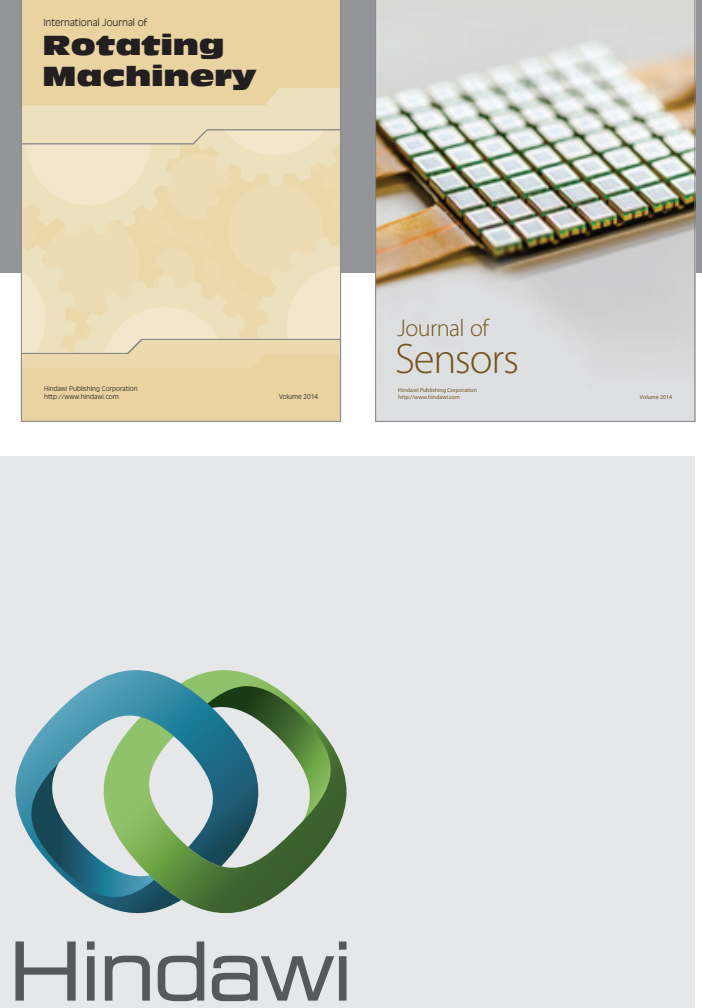

Submit your manuscripts at http://www.hindawi.com
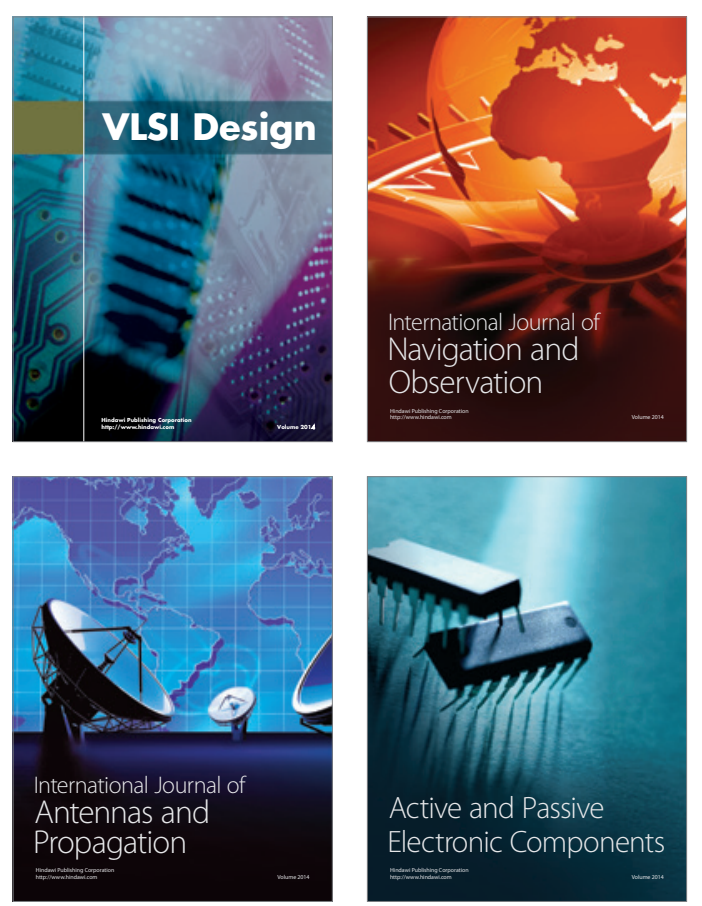
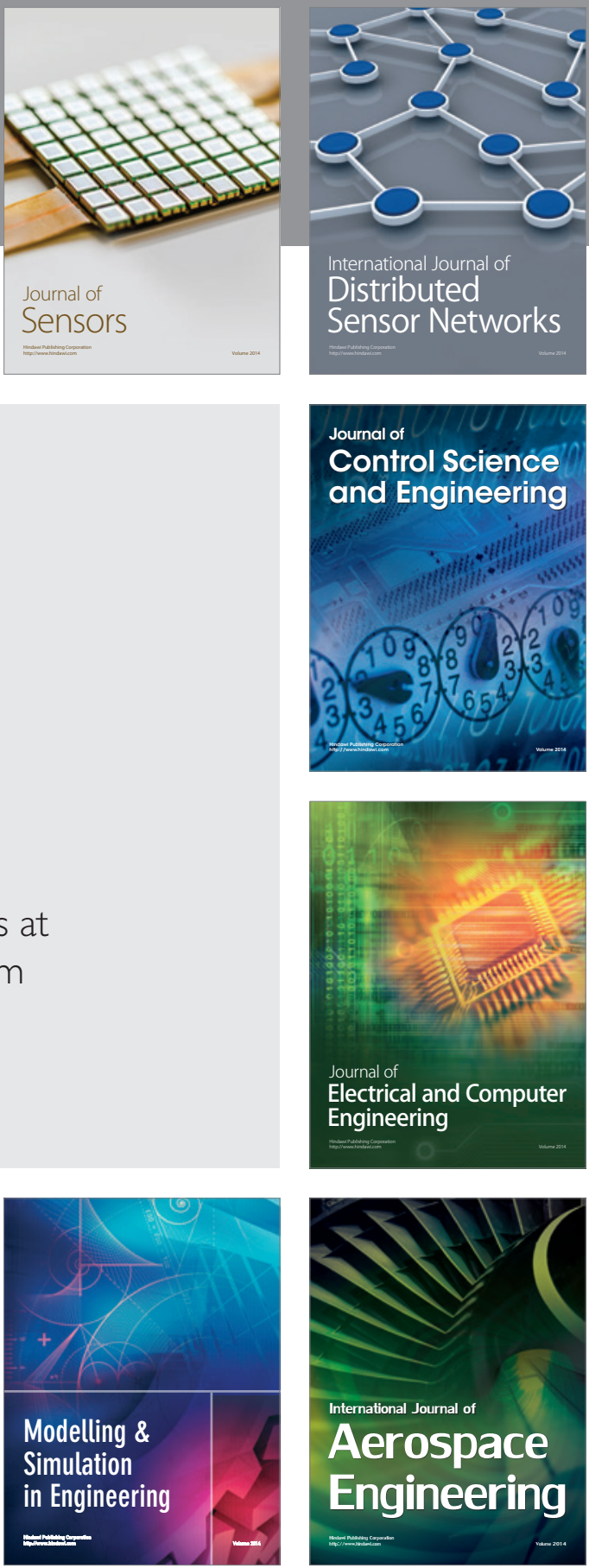

Journal of

Control Science

and Engineering
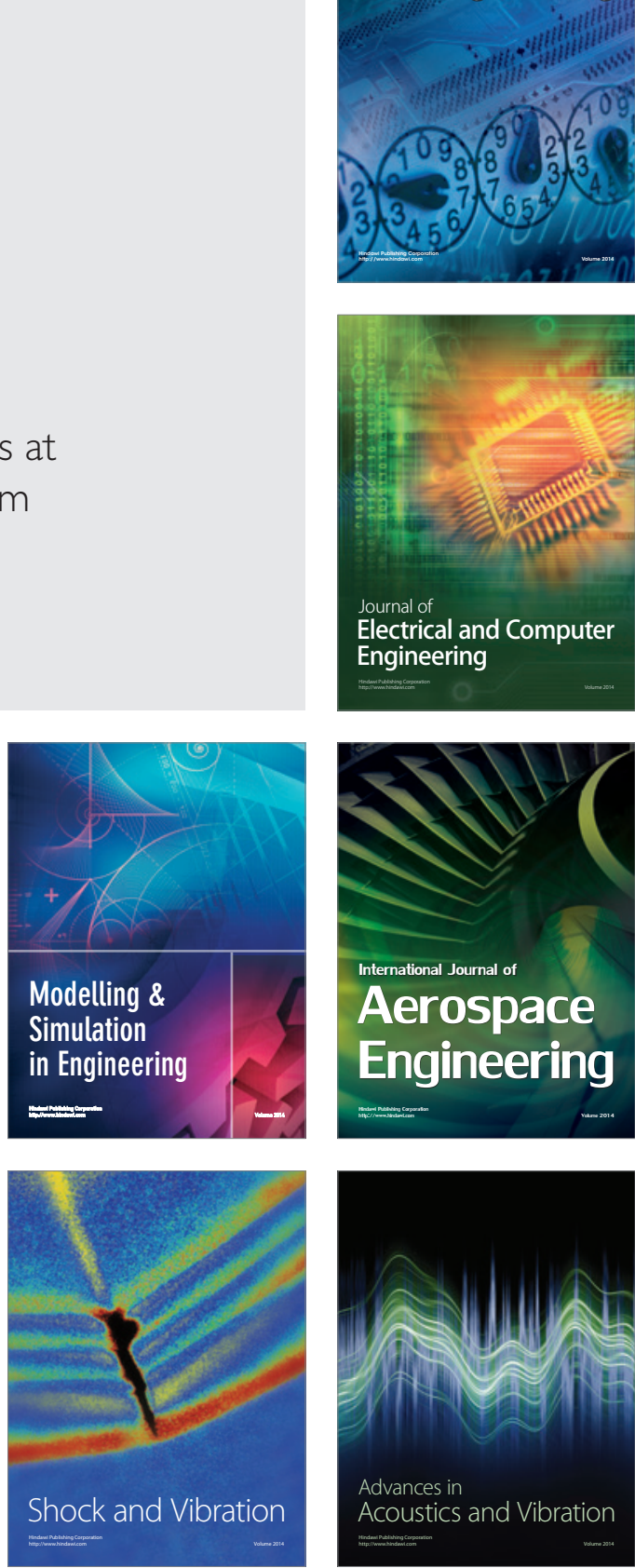